\title{
A microchannel flow application of a linearized kinetic Bhatnagar-Gross-Krook- type model for inert gas mixtures with general intermolecular forces
}

Cite as: Phys. Fluids 31, 072001 (2019); https://doi.org/10.1063/1.5098013

Submitted: 30 March 2019 . Accepted: 04 June 2019 . Published Online: 02 July 2019

Silvia Lorenzani (D)

\section{COLLECTIONS}

Paper published as part of the special topic on Direct Simulation Monte Carlo - The Legacy of Graeme A. Bird
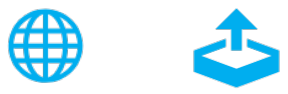

\section{ARTICLES YOU MAY BE INTERESTED IN}

Effective mean free path and viscosity of confined gases

Physics of Fluids 31, 072002 (2019); https://doi.org/10.1063/1.5108627

A micropolar-Newtonian blood flow model through a porous layered artery in the presence of a magnetic field

Physics of Fluids 31, 071901 (2019); https://doi.org/10.1063/1.5100802

A lattice Boltzmann method for simulating viscoelastic drops

Physics of Fluids 31, 073101 (2019); https://doi.org/10.1063/1.5100327

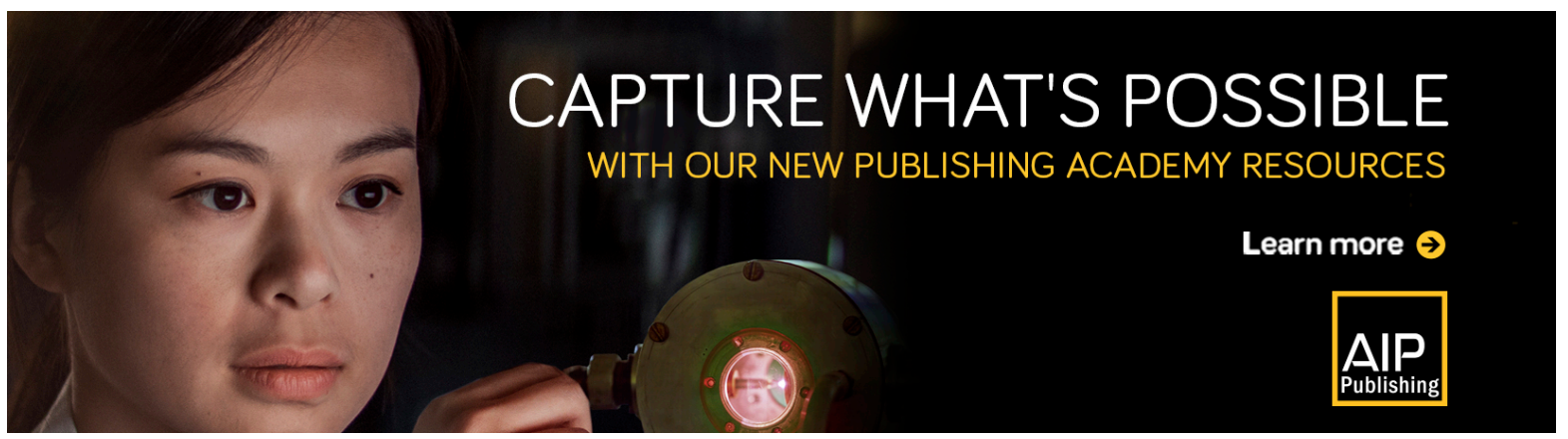




\title{
A microchannel flow application of a linearized kinetic Bhatnagar-Gross-Krook-type model for inert gas mixtures with general intermolecular forces
}

\author{
Cite as: Phys. Fluids 31, 072001 (2019); doi: 10.1063/1.5098013 \\ Submitted: 30 March 2019 • Accepted: 4 June 2019 • \\ Published Online: 2 July 2019
}

Silvia Lorenzani (iD)

\section{AFFILIATIONS}

Dipartimento di Matematica, Politecnico di Milano, Piazza Leonardo da Vinci 32, I-20133 Milano, Italy

Note: This paper is part of the special issue on Direct Simulation Monte Carlo-The Legacy of Graeme A. Bird.

\begin{abstract}
The flow of binary gaseous mixtures in microchannels, driven by a gradient of pressure, is investigated using the linearized Boltzmann equation based on a Bhatnagar-Gross-Krook-type model, able to describe general collision kernels, and diffuse reflection boundary conditions. Semi-analytical solutions have been obtained through a transformation in integral equations and the results compared with those derived by the McCormack model, which have revealed a good consistency with the experimental data.
\end{abstract}

Published under license by AIP Publishing. https://doi.org/10.1063/1.5098013

\section{INTRODUCTION}

In many practical problems, one is dealing not with pure gases but with gas mixtures. A wide range of applications include scientific and industrial processes. There are many gas mixtures used in clinical and medical practice (for patient diagnostics, to calibrate and maintain medical devices), in the food industry (to delay the deterioration of packaged food), and in manufacturing (for welding and cutting as well as for laser processing). ${ }^{1}$ With technological progress, the challenge has become that of integrating into a simple micro-sized system, operations that commonly solicit a whole laboratory. Microfluidic devices exploit the physical and chemical properties of liquids and gases at the microscale, offering several benefits over conventionally sized systems (i.e., portability, easier automation and parallelization, integration of lab routines in one device, and increase in measurement resolution). ${ }^{2}$ Since usually the characteristic length of such microdevices, operating in gaseous environments, is comparable with (or smaller than) the mean free path of the gas molecules, the gas cannot be treated as a continuous medium and the kinetic theory of rarefied gas flows in narrow channels must be applied.

The kinetic description of a mixture of gases with different particle masses (and possibly with different internal energies) is not a trivial generalization of the classical Boltzmann theory for a single gas since the collision operators have to take into account exchanges of momentum and energy among the different species (and also mass exchanges, in the case of reacting mixtures). Therefore, since it is difficult, in general, to manage the collision integral operator as such, simplified kinetic models have been proposed in the literature (see Refs. 4-13). Among them, the McCormack model ${ }^{8}$ has been, over the years, the most widely used to study a great variety of problems, such as Couette and Poiseuille flows, thermal creep, and heat transfer for mixtures, since all transport coefficients (i.e., viscosity, thermal conductivity, diffusion, and thermal diffusion ratio) can be correctly obtained from it by applying the Chapman-Enskog procedure. ${ }^{14-21}$ Moreover, McCormack derived a model for the crosscollision operator of a general multicomponent monatomic mixture applicable to different intermolecular force laws. Although the McCormack model provides a simplified description of a gas mixture compared to the Boltzmann true collision operator, it is still too complex to facilitate relatively simple analytical or semi-analytical manipulations. For monatomic gases, the model proposed by Bhatnagar, Gross, and Krook (BGK $)^{22}$ has revealed as a valuable tool in order to obtain approximate closed form solutions of the Boltzmann equation by using variational techniques or transformations in integral equations. ${ }^{23,24}$ BGK-type models for mixtures were first 
introduced in $1956,{ }^{4}$ and since then, many authors have focused their research on improving the mathematical consistency and predictability of these models compared to the full Boltzmann equation (see Refs. 5-7). In particular, the model proposed by Hamel, ${ }^{6}$ in which both self-collisions and cross-collisions return particles distributed according to a Maxwellian with an appropriate temperature and velocity, has recently been generalized in Ref. 12 to take into account interaction potentials different from the Maxwell molecules. The weak point of all these models is that they are characterized by many free parameters, the choice of which is far from obvious. In 2002, Andries, Aoki, and Perthame proposed in Ref. 9 a BGK relaxation model for mixtures that has been proven to be well posed from the mathematical point of view (correct Boltzmann collision invariants and Maxwellian equilibria are properly recovered, and the $\mathrm{H}$ theorem is fulfilled). The main idea behind this model is that instead of approximating each of the collision operators (between species $s$ and $r$ ) by a BGK-type equation (as in Refs. 6 and 12), only one global operator for each species $s$ (i.e., taking into account all the species $r$ ) has been introduced. As a result, the number of free parameters appearing in the model is significantly reduced. Even if the model presented in Ref. 9 has been derived in closedform by assuming Maxwell molecules and cannot match all the transport coefficients simultaneously, it has been used as a consistent tool in Ref. 25 to investigate the sound waves propagation in microchannels. Unfortunately, a more careful analysis has revealed that the results, although qualitatively correct, can show, in some cases, quantitative deviations from the predictions of the McCormack model, ${ }^{26}$ confirming that a correct description of the interparticle force law is of paramount importance to reproduce properly experimental results for gas mixtures. ${ }^{27}$

Consequently, in the present paper, we propose to modify the BGK model, introduced in Ref. 9, to make it able to describe general collision kernels corresponding to more realistic intermolecular potentials. Based on this modified BGK model, we report in the following the study of binary gas-mixture flows between parallel plates, driven by a pressure gradient, and diffuse reflection boundary conditions. These days, such a kind of investigation is no longer academic and deserves particular attention, thanks to the growing diffusion of microscale technologies. In this frame, an important aspect of the matter is to have an approximate closed form solution for the flow rate of plane Poiseuille flow in order to use it in applications. This need typically occurs in the correction of the Reynolds equation in lubrication theory or in modeling damping forces in microelectromechanical systems (MEMS) devices. ${ }^{28,29}$ In order to assess the reliability of the results obtained, we have compared the outputs of our semi-analytical method of solution with those deriving from the direct simulation Monte Carlo (DSMC) approach. This simulation scheme, which started with the ingenious work of Bird in the 1960s, ${ }^{30}$ has become, over the years, a powerful tool for solving practical physical problems, so much as to be considered an effective alternative to experiments. In particular, we rely on two different datasets: one obtained from a classical DSMC method, designed to solve the true linearized Boltzmann equation (LBE) for binary gas mixtures with hard-sphere molecules, ${ }^{31}$ and the other derived from a variance-reduced direct simulation Monte Carlo method, specific for low speed flows, as defined by the McCormack kinetic model. ${ }^{3}$ A further application of this modified BGK model can be found in Ref. 26.

\section{BOLTZMANN DESCRIPTION OF GASEOUS MIXTURES}

In kinetic theory, the evolution of a mixture of $N$ elastically scattering gases is usually described by a set of $N$ integrodifferential equations of Boltzmann type for the species distribution functions $f_{s}(t, \mathbf{x}, \boldsymbol{\xi})(s=1, \ldots, N)$,

$$
\frac{\partial f_{s}}{\partial t}+\boldsymbol{\xi} \cdot \nabla_{\mathbf{x}} f_{s}=\sum_{r=1}^{N} Q^{s r}\left(f_{s}, f_{r}\right)
$$

where the collision operator $Q^{s r}$ is given by

$Q^{s r}\left(f_{s}, f_{r}\right)=\int_{\mathbb{R}^{3}} \int_{B_{+}}\left[f_{s}\left(\xi^{\prime}\right) f_{r}\left(\xi_{*}^{\prime}\right)-f_{s}(\boldsymbol{\xi}) f_{r}\left(\boldsymbol{\xi}_{*}\right)\right] \mathcal{B}_{s r}(\mathbf{n} \cdot \mathbf{V},|\mathbf{V}|) d \boldsymbol{\xi}_{*} d \mathbf{n}$.

Here, $\boldsymbol{\xi}$ is the molecular velocity, $\mathcal{B}_{s r}$ is computed from the interaction law between the $s$ th and $r$ th species, $\mathbf{V}=\boldsymbol{\xi}-\boldsymbol{\xi}_{*}$ is the relative velocity of the molecule of the $s$ th species with respect to a molecule of the $r$ th species, $\mathbf{n}$ is an unit vector, and $B_{+}$is the semisphere defined by $\mathbf{n} \cdot \mathbf{V}=0$. The postcollisional velocities $\xi^{\prime}$ and $\boldsymbol{\xi}_{*}^{\prime}$ are given by

$$
\begin{aligned}
& \boldsymbol{\xi}^{\prime}=\boldsymbol{\xi}-\frac{2 \mu^{s r}}{m_{s}} \mathbf{n}\left[\left(\boldsymbol{\xi}-\boldsymbol{\xi}_{*}\right) \cdot \mathbf{n}\right], \\
& \boldsymbol{\xi}_{*}^{\prime}=\boldsymbol{\xi}_{*}+\frac{2 \mu^{s r}}{m_{r}} \mathbf{n}\left[\left(\boldsymbol{\xi}-\boldsymbol{\xi}_{*}\right) \cdot \mathbf{n}\right],
\end{aligned}
$$

where $\mu^{s r}=\frac{m_{s} m_{r}}{\left(m_{s}+m_{r}\right)}$ stands for the reduced mass. Since in microchannel flow applications, one usually deals with small deviations from the basic equilibrium state, the Boltzmann equations (1) and (2) can be linearized representing the distribution functions of the species as

$$
f_{s}=f_{s, 0}\left(1+h_{s}\right) \quad\left|h_{s}\right| \ll 1,
$$

with $f_{s, 0}$ being the Maxwellian configuration,

$$
f_{s, 0}=n_{s, 0}\left(\frac{m_{s}}{2 \pi k_{B} T_{0}}\right)^{3 / 2} \exp \left(-\frac{m_{s}}{2 k_{B} T_{0}} \xi^{2}\right)
$$

In (4), $k_{B}$ is the Boltzmann constant, $m_{s}$ and $n_{s, 0}$ are the mass and the equilibrium density of the sth species, and $T_{0}$ is a reference temperature. The small perturbation $h_{s}$ satisfies the following Boltzmann equation:

$$
\frac{\partial h_{s}}{\partial t}+\boldsymbol{\xi} \cdot \nabla_{\mathbf{x}} h_{s}=\sum_{r=1}^{N} L^{s r} h_{s}
$$

where $L^{s r} h_{s}$ is the linearized collision operator given by

$$
\begin{aligned}
L^{s r} h_{s}= & n_{r, 0}\left(\frac{m_{r}}{2 \pi k_{B} T_{0}}\right)^{3 / 2} \int_{\mathbb{R}^{3}} \int_{B_{+}}\left[h_{s}\left(\xi^{\prime}\right)+h_{r}\left(\xi_{*}^{\prime}\right)-h_{s}(\xi)-h_{r}\left(\xi_{*}\right)\right] \\
& \times \exp \left(-\frac{m_{r}}{2 k_{B} T_{0}} \xi_{*}^{2}\right) \cdot \mathcal{B}_{s r}(\mathbf{n} \cdot \mathbf{V},|\mathbf{V}|) d \boldsymbol{\xi}_{*} d \mathbf{n} .
\end{aligned}
$$

Since it is difficult, in general, to manage the collision integral operator as such, simplified kinetic models have been developed in the literature and widely used in practice. 


\section{A. McCormack model}

In 1973, McCormack proposed in Ref. 8 a simple method of construction of linearized kinetic models of the Boltzmann equation for gas mixtures. For the $k$ th-order model, the method consists of writing the collision term in the form

$$
\hat{L}_{(k)}^{s r} h_{s}=-\gamma^{s r} h_{s}(t, \mathbf{x}, \boldsymbol{\xi})+\sum_{j \leq k} A_{j}(t, \mathbf{x}) \psi_{j}(\boldsymbol{\xi}),
$$

where $\gamma^{s r}$ is a constant collision frequency and $\psi_{j}$ is a suitably chosen complete orthonormal set of functions. The expansion coefficients $A_{j}$ can be evaluated by equating certain physically significant moments of the model

$$
(\Delta \phi)_{s r}=\int \phi(\xi) f_{s, 0} \hat{L}_{(k)}^{s r} h_{s} d \xi
$$

to the corresponding moments of the full collision operator (6), calculated with the $k$ th-order approximation to the distribution function. For a third-order model, which is the lowest order that gives a correct hydrodynamic description, the collision term moments corresponding to the species density $(\phi=1)$, drift velocity $(\phi$ $\left.=\xi_{i}\right)$, energy $\left(\phi=\frac{1}{2} \xi^{2}\right)$, stress $\left(\phi=\xi_{i} \xi_{j}-\frac{1}{3} \xi^{2} \delta_{i j}\right)$, and heat flux $\left(\phi=\frac{1}{2} \xi_{i}\left(\xi^{2}-\frac{5}{2}\right)\right)$ have been computed by using a thirdorder, thirteen-moment approximation to the distribution function. Thus, the explicit McCormack collision term for a binary mixture reads

$$
\begin{aligned}
L h_{M C}^{(1)}= & \gamma_{1}\left\{\rho^{(1)}+2\left(1-\eta_{1,2}^{(1)}\right) \mathbf{c} \cdot \mathbf{v}^{(1)}-2 \eta_{1,2}^{(2)} \mathbf{c} \cdot \mathbf{q}^{(1)}+\left[1-\frac{2 \eta_{1,2}^{(1)} M_{12}}{\left(1+M_{12}\right)}\right]\left(|\mathbf{c}|^{2}-\frac{3}{2}\right) \tau^{(1)}\right. \\
& +2\left(1+\eta_{1,1}^{(4)}-\eta_{1,1}^{(3)}-\eta_{1,2}^{(3)}\right) c_{i} c_{j} \tilde{P}_{i j}^{(1)}+\frac{8}{5}\left(1+\eta_{1,1}^{(6)}-\eta_{1,1}^{(5)}-\eta_{1,2}^{(5)}\right)\left(|\mathbf{c}|^{2}-\frac{5}{2}\right) \mathbf{c} \cdot \mathbf{q}^{(1)} \\
& -\eta_{1,2}^{(2)}\left(|\mathbf{c}|^{2}-\frac{5}{2}\right) \mathbf{c} \cdot \mathbf{v}^{(1)}+2 \eta_{1,2}^{(1)} \mathbf{c} \cdot \mathbf{v}^{(2)}+2 M_{12} \eta_{1,2}^{(2)} \mathbf{c} \cdot \mathbf{q}^{(2)}+\eta_{1,2}^{(2)}\left(|\mathbf{c}|^{2}-\frac{5}{2}\right) \mathbf{c} \cdot \mathbf{v}^{(2)} \\
& \left.+\frac{2 \eta_{1,2}^{(1)} M_{12}}{\left(1+M_{12}\right)}\left(|\mathbf{c}|^{2}-\frac{3}{2}\right) \tau^{(2)}+\frac{2 \eta_{1,2}^{(4)}}{M_{12}} c_{i} c_{j} \tilde{P}_{i j}^{(2)}+\frac{8 \eta_{1,2}^{(6)}}{5 \sqrt{M_{12}}}\left(|\mathbf{c}|^{2}-\frac{5}{2}\right) \mathbf{c} \cdot \mathbf{q}^{(2)}-h_{1}\right\}, \\
L h^{(2)}{ }_{M C}= & \gamma_{2}\left\{\rho^{(2)}+\frac{2}{M_{12}}\left(1-\eta_{2,1}^{(1)}\right) \mathbf{c} \cdot \mathbf{v}^{(2)}-\frac{2 \eta_{2,1}^{(2)}}{M_{12}} \mathbf{c} \cdot \mathbf{q}^{(2)}+\left[1-\frac{2 \eta_{2,1}^{(1)}}{\left(1+M_{12}\right)}\right]\left(\frac{|\mathbf{c}|^{2}}{M_{12}}-\frac{3}{2}\right) \tau^{(2)}\right. \\
+ & \frac{2}{M_{12}^{2}}\left(1+\eta_{2,2}^{(4)}-\eta_{2,2}^{(3)}-\eta_{2,1}^{(3)}\right) c_{i} c_{j} \tilde{P}_{i j}^{(2)}+\frac{8}{5 M_{12}}\left(1+\eta_{2,2}^{(6)}-\eta_{2,2}^{(5)}-\eta_{2,1}^{(5)}\right)\left(\frac{|\mathbf{c}|^{2}}{M_{12}}-\frac{5}{2}\right) \mathbf{c} \cdot \mathbf{q}^{(2)} \\
- & \frac{\eta_{2,1}^{(2)}}{M_{12}}\left(\frac{|\mathbf{c}|^{2}}{M_{12}}-\frac{5}{2}\right) \mathbf{c} \cdot \mathbf{v}^{(2)}+\frac{2 \eta_{2,1}^{(1)}}{M_{12}} \mathbf{c} \cdot \mathbf{v}^{(1)}+\frac{2 \eta_{2,1}^{(2)}}{M_{12}^{2}} \mathbf{c} \cdot \mathbf{q}^{(1)}+\frac{\eta_{2,1}^{(2)}}{M_{12}}\left(\frac{|\mathbf{c}|^{2}}{M_{12}}-\frac{5}{2}\right) \mathbf{c} \cdot \mathbf{v}^{(1)} \\
+ & \left.\frac{2 \eta_{2,1}^{(1)}}{\left(1+M_{12}\right)}\left(\frac{|\mathbf{c}|^{2}}{M_{12}}-\frac{3}{2}\right) \tau^{(1)}+\frac{2 \eta_{2,1}^{(4)}}{M_{12}} c_{i} c_{j} \tilde{P}_{i j}^{(1)}+\frac{8 \eta_{2,1}^{(6)}}{5 \sqrt{M_{12}}}\left(\frac{|\mathbf{c}|^{2}}{M_{12}}-\frac{5}{2}\right) \mathbf{c} \cdot \mathbf{q}^{(1)}-h_{2}\right\},
\end{aligned}
$$

where it should be noted that, in combining the self- and crosscollision terms in (5), $\gamma$ 's appear only in the combinations: $\gamma_{1}=\gamma^{11}$ $+\gamma^{12}$ and $\gamma_{2}=\gamma^{21}+\gamma^{22}$. In (9) and (10), the following normalizations have been introduced:

$$
\mathbf{c}=\frac{\boldsymbol{\xi}}{\sqrt{2 \frac{k_{B}}{m_{1}} T_{0}}}, \quad \hat{f}_{1,0}=\frac{f_{1,0}}{n_{1,0}}=\frac{e^{-|\mathbf{c}|^{2}}}{\pi^{3 / 2}}, \quad \hat{f}_{2,0}=\frac{f_{2,0}}{n_{2,0}}=\frac{e^{-\frac{|c|^{2}}{M_{12}}}}{\left(\pi M_{12}\right)^{3 / 2}},
$$

where $M_{12}=m_{1} / m_{2}$ is the mass ratio. The other symbols appearing in (9) and (10) are defined in the following:

$$
\eta_{s, r}^{(k)}=\frac{v_{s, r}^{(k)}}{\gamma_{s}} \quad(s, r=1,2 \quad k=1, \ldots, 6),
$$

where the collision frequencies $\gamma_{s}(s=1,2)$ are expressed as ${ }^{15,17,18}$

$$
\begin{aligned}
& \gamma_{1}=\left(\psi_{1} \psi_{2}-v_{1,2}^{(4)} v_{2,1}^{(4)}\right)\left(\psi_{2}+v_{1,2}^{(4)}\right)^{-1}, \\
& \gamma_{2}=\left(\psi_{1} \psi_{2}-v_{1,2}^{(4)} v_{2,1}^{(4)}\right)\left(\psi_{1}+v_{2,1}^{(4)}\right)^{-1},
\end{aligned}
$$

with

$$
\psi_{1}=v_{1,1}^{(3)}+v_{1,2}^{(3)}-v_{1,1}^{(4)}, \quad \psi_{2}=v_{2,2}^{(3)}+v_{2,1}^{(3)}-v_{2,2}^{(4)}
$$

and

$$
v_{s, r}^{(1)}=\frac{16}{3} \frac{\mu^{s r}}{m_{s}} n_{r} \Omega_{s, r}^{11}, \quad v_{s, r}^{(2)}=\frac{64}{15}\left(\frac{\mu^{s r}}{m_{s}}\right)^{2} n_{r}\left(\Omega_{s, r}^{12}-\frac{5}{2} \Omega_{s, r}^{11}\right),
$$




$$
\begin{gathered}
v_{s, r}^{(3)}=\frac{16}{5}\left(\frac{\mu^{s r}}{m_{s}}\right)^{2} \frac{m_{s}}{m_{r}} n_{r}\left(\frac{10}{3} \Omega_{s, r}^{11}+\frac{m_{r}}{m_{s}} \Omega_{s, r}^{22}\right), \\
v_{s, r}^{(4)}=\frac{16}{5}\left(\frac{\mu^{s r}}{m_{s}}\right)^{2} \frac{m_{s}}{m_{r}} n_{r}\left(\frac{10}{3} \Omega_{s, r}^{11}-\Omega_{s, r}^{22}\right), \\
v_{s, r}^{(5)}=\frac{64}{15}\left(\frac{\mu^{s r}}{m_{s}}\right)^{3} \frac{m_{s}}{m_{r}} n_{r} \Gamma_{s, r}^{(5)}, \quad v_{s, r}^{(6)}=\frac{64}{15}\left(\frac{\mu^{s r}}{m_{s}}\right)^{3}\left(\frac{m_{s}}{m_{r}}\right)^{3 / 2} n_{r} \Gamma_{s, r}^{(6)},
\end{gathered}
$$

with

$$
\begin{gathered}
\Gamma_{s, r}^{(5)}=\Omega_{s, r}^{22}+\left(\frac{15 m_{s}}{4 m_{r}}+\frac{25 m_{r}}{8 m_{s}}\right) \Omega_{s, r}^{11}-\left(\frac{m_{r}}{2 m_{s}}\right)\left(5 \Omega_{s, r}^{12}-\Omega_{s, r}^{13}\right), \\
\Gamma_{s, r}^{(6)}=-\Omega_{s, r}^{22}+\frac{55}{8} \Omega_{s, r}^{11}-\frac{5}{2} \Omega_{s, r}^{12}+\frac{1}{2} \Omega_{s, r}^{13} .
\end{gathered}
$$

$\Omega_{s, r}^{i j}$ are the Chapman-Cowling integrals which are written in terms of the intermolecular interaction potential. ${ }^{14}$ The method to compute these integrals for several molecular models (considered in the present paper) is clarified in Appendix A.

Furthermore, the dimensionless macroscopic perturbed density $\left(\rho^{(s)}\right)$, velocity $\left(\mathbf{v}^{(s)}\right)$, temperature $\left(\tau^{(s)}\right)$, stress tensor $\left(\tilde{P}_{i j}^{(s)}\right)$, and heat flux $\left(\mathbf{q}^{(s)}\right)$, appearing in the McCormack model, are defined as follows:

$$
\rho^{(1)}=\frac{1}{\pi^{3 / 2}} \int_{\mathbf{R}^{3}} h_{1} \mathrm{e}^{-|\mathbf{c}|^{2}} d \mathbf{c}, \quad \rho^{(2)}=\frac{1}{\left(\pi M_{12}\right)^{3 / 2}} \int_{\mathbf{R}^{3}} h_{2} \mathrm{e}^{-\frac{|c|^{2}}{M_{12}}} d \mathbf{c},
$$

$$
\mathbf{v}^{(1)}=\frac{1}{\pi^{3 / 2}} \int_{\mathbf{R}^{3}} \mathbf{c} h_{1} \mathrm{e}^{-|\mathbf{c}|^{2}} d \mathbf{c}, \quad \mathbf{v}^{(2)}=\frac{1}{\left(\pi M_{12}\right)^{3 / 2}} \int_{\mathbf{R}^{3}} \mathbf{c} h_{2} \mathrm{e}^{-\frac{\left.|c|\right|^{2}}{M_{12}}} d \mathbf{c}
$$

$$
\begin{gathered}
\tau^{(1)}=\frac{1}{\pi^{3 / 2}} \int_{\mathbf{R}^{3}}\left(\frac{2}{3}|\mathbf{c}|^{2}-1\right) h_{1} \mathrm{e}^{-|\mathbf{c}|^{2}} d \mathbf{c}, \\
\tau^{(2)}=\frac{1}{\left(\pi M_{12}\right)^{3 / 2}} \int_{\mathbf{R}^{3}}\left(\frac{2}{3 M_{12}}|\mathbf{c}|^{2}-1\right) h_{2} \mathrm{e}^{-\frac{|\mathbf{c}|^{2}}{M_{12}}} d \mathbf{c}, \\
\tilde{P}_{i j}^{(1)}=\frac{1}{\pi^{3 / 2}} \int_{\mathbf{R}^{3}}\left(c_{i} c_{j}-\frac{1}{3}|\mathbf{c}|^{2} \delta_{i j}\right) h_{1} \mathrm{e}^{-|\mathbf{c}|^{2}} d \mathbf{c}, \\
\tilde{P}_{i j}^{(2)}=\frac{1}{\left(\pi M_{12}\right)^{3 / 2}} \int_{\mathbf{R}^{3}}\left(c_{i} c_{j}-\frac{1}{3}|\mathbf{c}|^{2} \delta_{i j}\right) h_{2} \mathrm{e}^{-\frac{|\mathbf{c}|^{2}}{M_{12}}} d \mathbf{c}, \\
\mathbf{q}^{(1)}=\frac{1}{\pi^{3 / 2}} \int_{\mathbf{R}^{3}} \frac{1}{2} \mathbf{c}\left(|\mathbf{c}|^{2}-\frac{5}{2}\right) h_{1} \mathrm{e}^{-|\mathbf{c}|^{2}} d \mathbf{c}, \\
\mathbf{q}^{(2)}=\frac{1}{\left(\pi M_{12}\right)^{3 / 2}} \int_{\mathbf{R}^{3}} \frac{1}{2} \mathbf{c}\left(\frac{|\mathbf{c}|^{2}}{M_{12}}-\frac{5}{2}\right) h_{2} \mathrm{e}^{-\frac{\left.|c|\right|^{2}}{M_{12}}} d \mathbf{c} .
\end{gathered}
$$

\section{B. BGK-type models}

In 2002, Andries, Aoki, and Perthame proposed in Ref. 9 a BGK relaxation model for mixtures which satisfies the following properties: the correct Boltzmann collision invariants and Maxwellian equilibria are properly recovered, the $\mathrm{H}$-theorem is fulfilled, and the indifferentiability principle holds (when the $N$ gases coincide, the classical BGK model for a single gas is correctly reproduced). For a mixture of two gases, this BGK model reads as

$$
\frac{\partial f_{s}}{\partial t}+\xi \cdot \nabla_{\mathrm{x}} f_{s}=v_{s}\left(\mathcal{M}_{s}-f_{s}\right), \quad s=1,2,
$$

where $v_{s}$ are suitable collision frequencies (independent from the molecular velocity $\xi$, but possibly dependent on the macroscopic fields) and $\mathcal{M}_{s}$ are Maxwellian attractors,

$$
\mathcal{M}_{s}=n_{s}\left(\frac{m_{s}}{2 \pi k_{B} T_{s}}\right)^{3 / 2} \exp \left[-\frac{m_{s}}{2 k_{B} T_{s}}\left|\boldsymbol{\xi}-\mathbf{v}_{s}\right|^{2}\right] .
$$

Auxiliary parameters $\mathbf{v}_{s}$ and $T_{s}$ are determined in terms of the moments of the distribution functions $f_{s}$, that is, the number density $n_{s}$, the mass velocity $\mathbf{v}^{(s)}$, and the temperature $T^{(s)}$, by imposing that the exchange rates for species momenta and energies given by the BGK operator reproduce the exact corresponding rates calculated by the Boltzmann collision operators $Q^{s r}\left(f_{s}, f_{r}\right)$ given by (2),

$$
\begin{aligned}
& v_{s} m_{s} \int_{\mathbb{R}^{3}} \xi\left[\mathcal{M}_{s}(\xi)-f_{s}(\xi)\right] d \xi=\sum_{r=1}^{2} m_{s} \int_{\mathbb{R}^{3}} \xi Q^{s r}\left(f_{s}, f_{r}\right) d \xi, \\
& v_{s} \frac{m_{s}}{2} \int_{\mathbb{R}^{3}}|\xi|^{2}\left[\mathcal{M}_{s}(\xi)-f_{s}(\xi)\right] d \xi=\sum_{r=1}^{2} \frac{m_{s}}{2} \int_{\mathbb{R}^{3}}|\xi|^{2} Q^{s r}\left(f_{s}, f_{r}\right) d \xi .
\end{aligned}
$$

The integrals on the right-hand side of Eqs. (27) and (28) have been computed in Ref. 9 by considering Maxwellian molecules since in this case, such rates can be made explicit in closed analytical form. Recent numerical works have revealed that, to reproduce properly experimental results for gas mixtures, a correct description of the interparticle force law is of paramount importance. ${ }^{17,20,32}$ Since the description of a mixture in terms of Maxwellian molecules is, on the contrary, highly unrealistic, in the present paper, we propose to modify the BGK model introduced in Ref. 9, by computing the integrals on the right-hand side of Eqs. (27) and (28) for general intermolecular forces with an approximation to the distribution function, similar to what was done in Ref. 8. In the framework of a linearized theory, when the distribution functions of the species $f_{s}$ can be represented as in (3) and (4), the constraints (27) and (28) give the following final expressions for the auxiliary fields appearing in (26):

$$
\begin{gathered}
\mathbf{v}_{1}=\mathbf{v}^{(1)}+\eta_{1,2}^{(1)}\left(\mathbf{v}^{(2)}-\mathbf{v}^{(1)}\right), \quad \mathbf{v}_{2}=\mathbf{v}^{(2)}+\eta_{2,1}^{(1)}\left(\mathbf{v}^{(1)}-\mathbf{v}^{(2)}\right), \\
T_{1}=T^{(1)}+\frac{2 m_{1}}{\left(m_{1}+m_{2}\right)} \eta_{1,2}^{(1)}\left(T^{(2)}-T^{(1)}\right), \\
T_{2}=T^{(2)}+\frac{2 m_{2}}{\left(m_{1}+m_{2}\right)} \eta_{2,1}^{(1)}\left(T^{(1)}-T^{(2)}\right),
\end{gathered}
$$


where the collision frequencies $\eta_{s, r}^{(k)}$ are the same as those defined in (12) after the identification $v_{s}=\gamma_{s}(s=1,2)$ and neglecting higher order terms,

$$
\begin{gathered}
\mathbf{v}^{(s)}=\frac{1}{n_{s, 0}} \int_{\mathbb{R}^{3}} \boldsymbol{\xi} f_{s, 0} h_{s} d \boldsymbol{\xi}, \\
T^{(s)}=T_{0}+\frac{m_{s}}{3 k_{B} n_{s, 0}} \int_{\mathbb{R}^{3}}\left(|\boldsymbol{\xi}|^{2}-\frac{3 k_{B}}{m_{s}} T_{0}\right) f_{s, 0} h_{s} d \boldsymbol{\xi} .
\end{gathered}
$$

In this way, a new BGK model can be constructed no longer restricted to Maxwell molecules but applicable to different and more realistic intermolecular force laws. Introducing the normalizations (11), the linearized BGK collision term can be written as

$$
\begin{aligned}
L h_{B G K}^{(1)}= & \gamma_{1}\left\{\rho^{(1)}+2\left(1-\eta_{1,2}^{(1)}\right) \mathbf{c} \cdot \mathbf{v}^{(1)}+2 \eta_{1,2}^{(1)} \mathbf{c} \cdot \mathbf{v}^{(2)}\right. \\
& +\left[1-\frac{2 \eta_{1,2}^{(1)} M_{12}}{\left(1+M_{12}\right)}\right]\left(|\mathbf{c}|^{2}-\frac{3}{2}\right) \tau^{(1)} \\
& \left.+\frac{2 \eta_{1,2}^{(1)} M_{12}}{\left(1+M_{12}\right)}\left(|\mathbf{c}|^{2}-\frac{3}{2}\right) \tau^{(2)}-h_{1}\right\} \\
L h_{B G K}^{(2)}= & \gamma_{2}\left\{\rho^{(2)}+2\left(1-\eta_{2,1}^{(1)}\right) \frac{\mathbf{c}}{M_{12}} \cdot \mathbf{v}^{(2)}+2 \eta_{2,1}^{(1)} \frac{\mathbf{c}}{M_{12}} \cdot \mathbf{v}^{(1)}\right. \\
+ & {\left[1-\frac{2 \eta_{2,1}^{(1)}}{\left(1+M_{12}\right)}\right]\left(\frac{|\mathbf{c}|^{2}}{M_{12}}-\frac{3}{2}\right) \tau^{(2)} } \\
+ & \left.\frac{2 \eta_{2,1}^{(1)}}{\left(1+M_{12}\right)}\left(\frac{|\mathbf{c}|^{2}}{M_{12}}-\frac{3}{2}\right) \tau^{(1)}-h_{2}\right\}
\end{aligned}
$$

where the dimensionless macroscopic perturbed density $\rho^{(s)}$, velocity $\mathbf{v}^{(s)}$, and temperature $\tau^{(s)}$ are defined in (17)-(20).

We will prove in the following that this modified BGK model is able to produce results in close agreement with those obtained with more refined kinetic models. The advantage is that the equations which describe the BGK model are much simpler than those which describe, for instance, the McCormack model, allowing also a semi-analytical representation of the solution.

\section{THE POISEUILLE FLOW PROBLEM: MATHEMATICAL FORMULATION}

Let us consider a binary gaseous mixture confined between two flat, infinite, and parallel plates located at $x^{\prime}=-d / 2$ and $x^{\prime}=d / 2$. Both boundaries are held at the same constant temperature. We assume that the mixture flows parallel to the plates, in the $z^{\prime}$ direction, due to a pressure gradient, which is taken to be small. Under these conditions, the Boltzmann equation can be linearized about local Maxwellian distributions by putting

$$
f_{s}\left(x^{\prime}, z^{\prime}, \boldsymbol{\xi}\right)=f_{s, 0}\left[1+k z^{\prime}+h_{s}\left(x^{\prime}, \boldsymbol{\xi}\right)\right], \quad s=1,2,
$$

where $f_{s, 0}$ is written explicitly in Eq. (4), $h_{s}$ is the small perturbation with respect to the equilibrium state, and $k=\frac{1}{p} \frac{\partial p}{\partial z^{\prime}}$ is a constant pressure gradient. Following Ref. 18, the system of linearized
Boltzmann equations reads as

$$
\xi_{x} \frac{\partial h_{s}}{\partial x^{\prime}}+k \xi_{z}=L h^{(s)}, \quad s=1,2,
$$

where $L h^{(s)}$ is the linearized collision operator. Since the numerical solution of Eq. (34) based on the McCormack kinetic model is reported in Ref. 18, in the sequel, we will restrict ourselves to the solution of Eq. (34) based on the modified BGK model introduced in Sec. II B. To this end, it is convenient to rescale all variables appearing in Eq. (34) as follows:

$$
\mathbf{c}=\frac{\xi}{v_{0}^{1}} ; \quad x=\frac{x^{\prime}}{v_{0}^{1} \theta_{1}} ; \quad z=\frac{z^{\prime}}{v_{0}^{1} \theta_{1}}, \quad \text { with } \quad \theta_{1}=\frac{1}{\gamma_{1}}, v_{0}^{1}=\sqrt{\frac{2 k_{B} T_{0}}{m_{1}}} .
$$

Furthermore, we define $\Theta_{12}=\frac{\theta_{1}}{\theta_{2}}=\frac{\gamma_{2}}{\gamma_{1}}$ and $\delta=d /\left(v_{0}^{1} \theta_{1}\right)$, which is the dimensionless distance between the channel walls as well as the rarefaction parameter (inverse Knudsen number) of the species $s=1$. The unusual choice of normalizing the molecular velocities with respect to the thermal velocity of the species 1 depends on the fact that, in this way, it becomes more evident to identify in the equations written below, for the components of the mixture, the deviations from the single gas behavior, in terms of peculiar parameters such as the molecular mass ratio $M_{12}$, the macroscopic collision frequencies ratio $\Theta_{12}$, and the microscopic collision frequencies $\eta_{s, r}^{(k)}$.

Since the problem under consideration is one-dimensional in space, the unknown perturbed distribution functions $h_{s}$, as well as the overall quantities, depend only on the $x$ coordinate. Likewise, we can reduce the dimensionality of the molecular-velocity space by introducing the projection procedure. ${ }^{34}$ We multiply Eq. (34) [based on the BGK-collision operator as defined in Eqs. (31) and (32)] by $\frac{1}{\pi} c_{z} e^{-\left(c_{y}^{2}+c_{z}^{2}\right)}$, when $s=1$, and by $\frac{1}{\pi M_{12}^{3 / 2}} c_{z} e^{-\left(c_{y}^{2}+c_{z}^{2}\right) / M_{12}}$, when $s=2$, and we integrate over all $c_{y}$ and $c_{z}$. The resulting equations after the projection are

$$
c_{x} \frac{\partial Z^{(1)}}{\partial x}+Z^{(1)}+1=\left(1-\eta_{1,2}^{(1)}\right) v_{z}^{(1)}+\eta_{1,2}^{(1)} v_{z}^{(2)},
$$

$$
\begin{aligned}
& c_{x} \frac{\partial Z^{(2)}}{\partial x}+\Theta_{12} Z^{(2)}+\sqrt{M_{12}} \\
& \quad=\Theta_{12}\left[\frac{1}{\sqrt{M_{12}}}\left(1-\eta_{2,1}^{(1)}\right) v_{z}^{(2)}+\frac{\eta_{2,1}^{(1)}}{\sqrt{M_{12}}} v_{z}^{(1)}\right],
\end{aligned}
$$

where the reduced unknown distribution functions $Z^{(s)}$ are defined as

$$
Z^{(1)}\left(x, c_{x}\right)=\frac{2}{\pi \hat{k}} \int_{-\infty}^{+\infty} \int_{-\infty}^{+\infty} h_{1}(x, \mathbf{c}) c_{z} e^{-\left(c_{y}^{2}+c_{z}^{2}\right)} d c_{y} d c_{z},
$$

$$
Z^{(2)}\left(x, c_{x}\right)=\frac{2}{\pi \hat{k} M_{12}^{3 / 2}} \int_{-\infty}^{+\infty} \int_{-\infty}^{+\infty} h_{2}(x, \mathbf{c}) c_{z} e^{-\left(c_{y}^{2}+c_{z}^{2}\right) / M_{12}} d c_{y} d c_{z}
$$


with $\hat{k}=\frac{1}{p} \frac{\partial p}{\partial z}$ being the dimensionless constant pressure gradient. The macroscopic velocity fields appearing on the right-hand side of Eqs. (35) and (36) are given by

$$
\begin{aligned}
& v_{z}^{(1)}(x)=\frac{1}{\sqrt{\pi}} \int_{-\infty}^{+\infty} Z^{(1)} e^{-c_{x}^{2}} d c_{x}, \\
& v_{z}^{(2)}(x)=\frac{1}{\sqrt{\pi}} \int_{-\infty}^{+\infty} Z^{(2)} e^{-c_{x}^{2} / M_{12}} d c_{x} .
\end{aligned}
$$

Applying the same projection procedure to the linearized boundary conditions reported in Ref. 18 describing the diffuse scattering of gaseous particles on both walls of the microchannel, we are led to derive the following expressions:

$$
\begin{aligned}
& Z^{(1)}\left(x=-\frac{\delta}{2}, c_{x}\right)=Z^{(2)}\left(x=-\frac{\delta}{2}, c_{x}\right)=0, \quad c_{x}>0, \\
& Z^{(1)}\left(x=\frac{\delta}{2}, c_{x}\right)=Z^{(2)}\left(x=\frac{\delta}{2}, c_{x}\right)=0, \quad c_{x}<0 .
\end{aligned}
$$

In addition to the bulk velocities of the gas components, we intend to compute the mass-flow rates defined for each species by

$$
\begin{gathered}
Q^{(1)}=-\frac{1}{\delta^{2}} \int_{-\delta / 2}^{\delta / 2} v_{z}^{(1)}(x) d x, \\
Q^{(2)}=-\frac{1}{\sqrt{M_{12}} \delta^{2}} \int_{-\delta / 2}^{\delta / 2} v_{z}^{(2)}(x) d x .
\end{gathered}
$$

\section{A. Semi-analytical solutions in integral form}

Integrating Eqs. (35) and (36) along the trajectories of the molecules with the boundary conditions (41) and (42), we get the solutions in integral form,

$$
\begin{aligned}
Z^{(1)}\left(x, c_{x}\right)=\frac{1}{c_{x}} & \int_{-\frac{\delta}{2} \operatorname{sgn} c_{x}}^{x}\left[-1+\left(1-\eta_{1,2}^{(1)}\right) v_{z}^{(1)}+\eta_{1,2}^{(1)} v_{z}^{(2)}\right] e^{-\frac{|x-t|}{\left|c_{x}\right|} d t} d t \\
Z^{(2)}\left(x, c_{x}\right)= & \frac{1}{c_{x}} \int_{-\frac{\delta}{2} \operatorname{sgn} c_{x}}^{x}\left[-\sqrt{M_{12}}+\frac{\Theta_{12}}{\sqrt{M_{12}}}\left(1-\eta_{2,1}^{(1)}\right) v_{z}^{(2)}\right. \\
& \left.+\frac{\Theta_{12}}{\sqrt{M_{12}}} \eta_{2,1}^{(1)} v_{z}^{(1)}\right] e^{-\Theta_{12} \frac{|x-t|}{\left|c_{x}\right|} d t .}
\end{aligned}
$$

Inserting in the definitions (39) and (40) the distribution functions (45) and (46), the integral equations for the bulk velocities of the gas components read as follows:

$$
\begin{aligned}
v_{z}^{(1)}(x)= & -1+\frac{1}{\sqrt{\pi}}\left[T_{0}\left(\frac{\delta}{2}+x\right)+T_{0}\left(\frac{\delta}{2}-x\right)\right] \\
& +\frac{\left(1-\eta_{1,2}^{(1)}\right)}{\sqrt{\pi}} \int_{-\delta / 2}^{\delta / 2} v_{z}^{(1)}(t) T_{-1}(|x-t|) d t \\
& +\frac{\eta_{1,2}^{(1)}}{\sqrt{\pi}} \int_{-\delta / 2}^{\delta / 2} v_{z}^{(2)}(t) T_{-1}(|x-t|) d t,
\end{aligned}
$$

$$
\begin{aligned}
v_{z}^{(2)}(x)= & -\frac{M_{12}}{\Theta_{12}}+\frac{M_{12}}{\sqrt{\pi} \Theta_{12}}\left\{T_{0}\left[\frac{\Theta_{12}}{\sqrt{M_{12}}}\left(\frac{\delta}{2}+x\right)\right]\right. \\
& \left.+T_{0}\left[\frac{\Theta_{12}}{\sqrt{M_{12}}}\left(\frac{\delta}{2}-x\right)\right]\right\}+\frac{\Theta_{12}}{\sqrt{\pi M_{12}}}\left(1-\eta_{2,1}^{(1)}\right) \\
& \times \int_{-\delta / 2}^{\delta / 2} v_{z}^{(2)}(t) T_{-1}\left[\frac{\Theta_{12}}{\sqrt{M_{12}}}|x-t|\right] d t+\frac{\Theta_{12}}{\sqrt{\pi M_{12}}} \eta_{2,1}^{(1)} \\
& \times \int_{-\delta / 2}^{\delta / 2} v_{z}^{(1)}(t) T_{-1}\left[\frac{\Theta_{12}}{\sqrt{M_{12}}}|x-t|\right] d t
\end{aligned}
$$

where $T_{n}$ denotes the Abramowitz functions defined by

$$
T_{n}(x):=\int_{0}^{+\infty} s^{n} e^{-s^{2}-\frac{x}{s}} d s .
$$

In order to solve the system of two coupled integral equations (47) and (48), we extend a finite difference technique introduced in Refs. 35, 36, and 24. The one-dimensional computational domain is divided into $n$ mesh points (for simplicity, only constant mesh steps are considered), and the macroscopic fields $\left(v_{z}^{(1)}, v_{z}^{(2)}\right)$ are approximated by a stepwise function. The general form of the numerical scheme is given by

$$
\sum_{k=0}^{2 n-1} \alpha_{h k} \psi_{k}=\beta_{h} \quad(h=0, \ldots, 2 n-1),
$$

where

$$
\begin{array}{rlrl}
\psi_{i} & =v_{z}^{(1)}\left(x_{i}\right) & & (i=0, \ldots, n-1), \\
\psi_{i+n} & =v_{z}^{(2)}\left(x_{i}\right) & (i=0, \ldots, n-1) .
\end{array}
$$

Following the idea reported in Ref. 24, the constant value assigned to the functions $v_{z}^{(1)}(x)$ and $v_{z}^{(2)}(x)$ on every interval can be interpreted as either (a) the value in the midpoint or (b) the mean value on the whole interval, so that two methods of differencing can be defined with two possible choices for the coefficients $\alpha_{h k}$ and $\beta_{h}$. In the following, we report only the coefficients related to the method of differencing (a) (which can be computed more easily) since with a resolution of $n=200$ mesh points (used in the present computations to reach very high accuracy), the two schemes approach so closely each other that they can be considered equivalent. The detailed form of the coefficients appearing in the scheme (50) is given by

$$
\begin{gathered}
\alpha_{h k}=\frac{\left(1-\eta_{1,2}^{(1)}\right)}{\sqrt{\pi}}\left[T_{0}\left(\frac{|k-h|}{n} \delta+\frac{\delta}{2 n}\right)-T_{0}\left(\frac{|k-h|}{n} \delta-\frac{\delta}{2 n}\right)\right] \\
\alpha_{k k}=\eta_{1,2}^{(1)}+\frac{2}{\sqrt{\pi}}\left(1-\eta_{1,2}^{(1)}\right) T_{0}\left(\frac{\delta}{2 n}\right) \\
\alpha_{h, k+n}=\frac{\eta_{1,2}^{(1)}}{\sqrt{\pi}}\left[T_{0}\left(\frac{|k-h|}{n} \delta+\frac{\delta}{2 n}\right)-T_{0}\left(\frac{|k-h|}{n} \delta-\frac{\delta}{2 n}\right)\right] \\
\alpha_{k, k+n}=-\eta_{1,2}^{(1)}+\frac{2 \eta_{1,2}^{(1)}}{\sqrt{\pi}} T_{0}\left(\frac{\delta}{2 n}\right)
\end{gathered}
$$




$$
\begin{aligned}
\alpha_{h+n, k}= & \frac{\eta_{2,1}^{(1)}}{\sqrt{\pi}}\left\{T_{0}\left[\frac{\Theta_{12}}{\sqrt{M_{12}}}\left(\frac{|k-h|}{n} \delta+\frac{\delta}{2 n}\right)\right]\right. \\
& \left.-T_{0}\left[\frac{\Theta_{12}}{\sqrt{M_{12}}}\left(\frac{|k-h|}{n} \delta-\frac{\delta}{2 n}\right)\right]\right\} \quad(h \neq k), \\
\alpha_{k+n, k}= & -\eta_{2,1}^{(1)}+\frac{2 \eta_{2,1}^{(1)}}{\sqrt{\pi}} T_{0}\left(\frac{\Theta_{12}}{\sqrt{M_{12}}} \frac{\delta}{2 n}\right),
\end{aligned}
$$

$$
\begin{aligned}
\alpha_{h+n, k+n}= & \frac{\left(1-\eta_{2,1}^{(1)}\right)}{\sqrt{\pi}}\left\{T_{0}\left[\frac{\Theta_{12}}{\sqrt{M_{12}}}\left(\frac{|k-h|}{n} \delta+\frac{\delta}{2 n}\right)\right]\right. \\
- & \left.T_{0}\left[\frac{\Theta_{12}}{\sqrt{M_{12}}}\left(\frac{|k-h|}{n} \delta-\frac{\delta}{2 n}\right)\right]\right\} \quad(h \neq k), \\
\alpha_{k+n, k+n} & =\eta_{2,1}^{(1)}+\frac{2}{\sqrt{\pi}}\left(1-\eta_{2,1}^{(1)}\right) T_{0}\left(\frac{\Theta_{12}}{\sqrt{M_{12}}} \frac{\delta}{2 n}\right),
\end{aligned}
$$

\begin{tabular}{|c|c|c|c|c|c|c|c|c|}
\hline & \multirow[b]{2}{*}{$\delta^{*}$} & \multicolumn{2}{|c|}{ DSMC (LBE H-S) } & \multicolumn{2}{|c|}{ DSMC (McC H-S) } & \multicolumn{2}{|c|}{ BGK (hard spheres) } & \multirow[b]{2}{*}{$\Delta_{\max }(\%)$} \\
\hline & & $Q^{(1)}$ & $Q^{(2)}$ & $Q^{(1)}$ & $Q^{(2)}$ & $Q^{(1)}$ & $Q^{(2)}$ & \\
\hline \multirow{6}{*}{$\mathrm{Ne}-\mathrm{Ar}$} & 0.1 & 1.981 & 1.932 & 2.0350 & 2.0531 & 2.0259 & 2.0576 & 0.4 \\
\hline & 0.5 & $\ldots$ & $\ldots$ & 1.5303 & 1.7019 & 1.5097 & 1.7019 & 1.3 \\
\hline & 1.0 & 1.385 & 1.632 & 1.4198 & 1.6863 & 1.3974 & 1.6833 & 1.6 \\
\hline & 2.0 & $\ldots$ & $\ldots$ & 1.4166 & 1.7987 & 1.3951 & 1.7932 & 1.5 \\
\hline & 5.0 & $\ldots$ & $\ldots$ & 1.6989 & 2.3007 & 1.6794 & 2.2901 & 1.1 \\
\hline & 10.0 & 2.271 & 3.151 & 2.3163 & 3.2122 & 2.3016 & 3.2027 & 0.6 \\
\hline \multirow{6}{*}{$\mathrm{He}-\mathrm{Xe}$} & 0.1 & 2.097 & 2.013 & 2.0608 & 2.2266 & 2.0414 & 2.2400 & 0.9 \\
\hline & 0.5 & $\ldots$ & $\ldots$ & 1.3607 & 1.9637 & 1.3225 & 1.9798 & 2.8 \\
\hline & 1.0 & 1.117 & 1.956 & 1.1117 & 2.0200 & 1.0701 & 2.0351 & 3.7 \\
\hline & 2.0 & $\ldots$ & $\ldots$ & 0.9145 & 2.2394 & 0.8762 & 2.2486 & 4.2 \\
\hline & 5.0 & $\ldots$ & $\ldots$ & 0.7914 & 2.9529 & 0.7645 & 2.9482 & 3.4 \\
\hline & 10.0 & 0.8584 & 4.040 & 0.8723 & 4.1184 & 0.8543 & 4.1063 & 2.1 \\
\hline
\end{tabular}

TABLE I. Poiseuille flow rate $Q^{(s)}$ vs $\delta^{*}$ for $N_{12}=1$. Comparison between the outputs obtained by the modified BGK model and those derived from the DSMC simulations designed to solve the McCormack model (McC H-S $)^{32}$ and the linearized Boltzmann equation (LBE H-S), ${ }^{31}$ for hard-sphere interactions. $\Delta_{\max }$ [defined in Eq. (64)] indicates the maximum absolute deviation of the results of the BGK model from those obtained by the McCormack one.

\begin{tabular}{|c|c|c|c|c|c|c|c|c|c|}
\hline & \multirow[b]{2}{*}{$\delta^{*}$} & \multicolumn{2}{|c|}{ BGK (Maxwell) } & \multirow[b]{2}{*}{$\Delta_{\max }(\%)$} & \multicolumn{2}{|c|}{ DSMC (McC H-S) } & \multicolumn{2}{|c|}{ BGK (hard spheres) } & \multirow[b]{2}{*}{$\Delta_{\max }(\%)$} \\
\hline & & $Q^{(1)}$ & $Q^{(2)}$ & & $Q^{(1)}$ & $Q^{(2)}$ & $Q^{(1)}$ & $Q^{(2)}$ & \\
\hline \multirow{6}{*}{$\mathrm{Ne}-\mathrm{Ar}$} & 0.1 & 2.0312 & 2.0835 & 0.2 & 2.0358 & 2.0796 & 2.0307 & 2.0908 & 0.5 \\
\hline & 0.5 & 1.5822 & 1.7949 & 1.2 & 1.5916 & 1.8164 & 1.5787 & 1.8283 & 0.8 \\
\hline & 1.0 & 1.5076 & 1.8229 & 1.6 & 1.5185 & 1.8521 & 1.5038 & 1.8603 & 0.9 \\
\hline & 2.0 & 1.5494 & 1.9947 & 1.6 & 1.5635 & 2.0274 & 1.5464 & 2.0283 & 1.0 \\
\hline & 5.0 & 1.9170 & 2.6140 & 1.0 & 1.9325 & 2.6416 & 1.9159 & 2.6353 & 0.8 \\
\hline & 10.0 & 2.6551 & 3.6938 & 0.4 & 2.6636 & 3.7078 & 2.6553 & 3.7069 & 0.3 \\
\hline \multirow{6}{*}{$\mathrm{He}-\mathrm{Xe}$} & 0.1 & 2.0774 & 2.6214 & 2.0 & 2.1191 & 2.6182 & 2.1092 & 2.6685 & 1.9 \\
\hline & 0.5 & 1.5312 & 2.7356 & 3.9 & 1.5470 & 2.8470 & 1.5252 & 2.9360 & 3.0 \\
\hline & 1.0 & 1.3794 & 3.1183 & 4.9 & 1.3805 & 3.2787 & 1.3552 & 3.3854 & 3.2 \\
\hline & 2.0 & 1.2989 & 3.8473 & 4.9 & 1.2866 & 4.0473 & 1.2605 & 4.1501 & 2.5 \\
\hline & 5.0 & 1.3695 & 5.6880 & 3.4 & 1.3486 & 5.8876 & 1.3272 & 5.9469 & 1.6 \\
\hline & 10.0 & 1.6763 & 8.3047 & 1.8 & 1.6594 & 8.4565 & 1.6423 & 8.4775 & 1.0 \\
\hline
\end{tabular}

TABLE II. Poiseuille flow rate $Q^{(s)}$ vs $\delta^{*}$ for $N_{12}=9$. Comparison between the results obtained by the BGK models for Maxwell molecules and modified for hard-sphere interactions and those derived from the DSMC simulations designed to solve the McCormack model for rigid spheres. ${ }^{32} \Delta_{\max }$ [defined in Eq. (64)] indicates the maximum absolute deviation of the outputs of each BGK model from those obtained by the McCormack one. 
TABLE III. Poiseuille flow rate $Q^{(s)}$ vs $\delta^{*}$ for $N_{12}=0.11$. Comparison between the results obtained by the BGK models for Maxwell molecules and modified for hard-sphere interactions and those derived from the DSMC simulations designed to solve the McCormack model for rigid spheres. ${ }^{32} \Delta_{\max }$ [defined in Eq. (64)] indicates the maximum absolute deviation of the outputs of each BGK model from those obtained by the McCormack one.

\begin{tabular}{|c|c|c|c|c|c|c|c|c|c|}
\hline & \multirow[b]{2}{*}{$\delta^{*}$} & \multicolumn{2}{|c|}{ BGK (Maxwell) } & \multirow[b]{2}{*}{$\Delta_{\max }(\%)$} & \multicolumn{2}{|c|}{ DSMC (McC H-S) } & \multicolumn{2}{|c|}{ BGK (hard spheres) } & \multirow[b]{2}{*}{$\Delta_{\max }(\%)$} \\
\hline & & $Q^{(1)}$ & $Q^{(2)}$ & & $Q^{(1)}$ & $Q^{(2)}$ & $Q^{(1)}$ & $Q^{(2)}$ & \\
\hline \multirow{6}{*}{$\mathrm{Ne}-\mathrm{Ar}$} & 0.1 & 2.0377 & 2.0352 & 0.2 & 2.0374 & 2.0391 & 2.0227 & 2.0368 & 0.7 \\
\hline & 0.5 & 1.4915 & 1.6154 & 0.8 & 1.4888 & 1.6278 & 1.4636 & 1.6184 & 1.7 \\
\hline & 1.0 & 1.3536 & 1.5596 & 1.0 & 1.3523 & 1.5752 & 1.3246 & 1.5628 & 2.0 \\
\hline & 2.0 & 1.3136 & 1.6254 & 1.0 & 1.3158 & 1.6421 & 1.2888 & 1.6283 & 2.0 \\
\hline & 5.0 & 1.5240 & 2.0400 & 0.7 & 1.5311 & 2.0556 & 1.5095 & 2.0418 & 1.4 \\
\hline & 10.0 & 2.0572 & 2.8415 & 0.6 & 2.0679 & 2.8581 & 2.0493 & 2.8426 & 0.9 \\
\hline \multirow{6}{*}{$\mathrm{He}-\mathrm{Xe}$} & 0.1 & 2.0640 & 2.0564 & 3.2 & 1.9993 & 2.0654 & 1.9741 & 2.0634 & 1.3 \\
\hline & 0.5 & 1.3426 & 1.6423 & 6.3 & 1.2631 & 1.6611 & 1.2190 & 1.6542 & 3.5 \\
\hline & 1.0 & 1.0773 & 1.5925 & 8.3 & 0.9949 & 1.6159 & 0.9489 & 1.6058 & 4.6 \\
\hline & 2.0 & 0.8547 & 1.6683 & 10. & 0.7775 & 1.6943 & 0.7370 & 1.6815 & 5.2 \\
\hline & 5.0 & 0.6743 & 2.1059 & 8.9 & 0.6194 & 2.1301 & 0.5934 & 2.1156 & 4.2 \\
\hline & 10.0 & 0.6857 & 2.9366 & 5.1 & 0.6523 & 2.9578 & 0.6358 & 2.9425 & 2.5 \\
\hline
\end{tabular}

$$
\begin{aligned}
\beta_{h}=-1+ & \frac{1}{\sqrt{\pi}}\left[T_{0}\left((2 h+1) \frac{\delta}{2 n}\right)+T_{0}\left(\delta-(2 h+1) \frac{\delta}{2 n}\right)\right], \\
\beta_{h+n}= & -\frac{M_{12}}{\Theta_{12}}+\frac{M_{12}}{\sqrt{\pi} \Theta_{12}}\left\{T_{0}\left[\frac{\Theta_{12}}{\sqrt{M_{12}}}\left((2 h+1) \frac{\delta}{2 n}\right)\right]\right. \\
& \left.+T_{0}\left[\frac{\Theta_{12}}{\sqrt{M_{12}}}\left(\delta-(2 h+1) \frac{\delta}{2 n}\right)\right]\right\},
\end{aligned}
$$

where $h, k=0,1, \ldots n-1$.

\section{RESULTS AND DISCUSSION}

The results presented in the following refer to the noble gaseous mixtures of $\mathrm{He}-\mathrm{Xe}$ (that is, helium with molecular mass $m_{1}=4.0026$ a.u. and xenon with molecular mass $m_{2}=131.29$ a.u.) and $\mathrm{Ne}-\mathrm{Ar}$ (that is, neon with molecular mass $m_{1}=20.179$ a.u. and argon with molecular mass $m_{2}=39.948$ a.u.). Thus, the present study includes mixtures whose constituents have comparable molecular mass as well as disparate-mass gas mixtures (composed of very heavy plus very light molecules). In order to assess the reliability of the results based on the modified BGK model proposed in Sec. II B, we specialize the analysis to the case of rigid-sphere interactions and compare our findings with those obtained by using the direct simulation Monte Carlo method (DSMC). In particular, we rely on the outputs from the standard DSMC method developed to solve the linearized Boltzmann equation for binary gas mixtures ${ }^{31}$ and those from a variance-reduced DSMC methodology presented for the solution of the McCormack linearized kinetic model. ${ }^{32}$ In our computations, the rigid-sphere diameters $d_{s}(s=1,2)$ of every species $s$ are determined as indicated in Appendix A. Thus, the diameter ratios $D_{12}=d_{1} / d_{2}$ are taken equal to 0.71097 and 0.4492 for the mixtures $\mathrm{Ne}-\mathrm{Ar}$ and $\mathrm{He}-\mathrm{Xe}$, respectively. In Tables I-III, we list the values of
TABLE IV. Poiseuille flow rate $Q^{(s)}$ vs $\delta^{*}$ for the He-Xe mixture. Comparison between the results obtained by the modified BGK model for the (6-12) LennardJones (LJ) interaction potential and those derived from the McCormack model with the same intermolecular force, for three different values of the ratio of the molar concentrations of the two species $N_{12} . \Delta_{\max }$ [defined in Eq. (64)] indicates the maxi-

\begin{tabular}{|c|c|c|c|c|c|c|}
\hline & \multirow[b]{2}{*}{$\delta^{*}$} & \multicolumn{2}{|c|}{$\operatorname{McC}(\mathrm{LJ})$} & \multicolumn{2}{|c|}{ BGK (LJ) } & \multirow[b]{2}{*}{$\Delta_{\max }(\%)$} \\
\hline & & $Q^{(1)}$ & $Q^{(2)}$ & $Q^{(1)}$ & $Q^{(2)}$ & \\
\hline \multirow{6}{*}{$\mathrm{N}_{12}=1$} & 0.1 & 2.1411 & 2.1932 & 2.1268 & 2.1994 & 0.7 \\
\hline & 0.5 & 1.4279 & 1.9216 & 1.4017 & 1.9239 & 1.8 \\
\hline & 1.0 & 1.1712 & 1.9761 & 1.1421 & 1.9760 & 2.5 \\
\hline & 2.0 & 0.9642 & 2.2001 & 0.9361 & 2.1938 & 2.9 \\
\hline & 5.0 & 0.8241 & 2.9310 & 0.8029 & 2.9155 & 2.6 \\
\hline & 10.0 & 0.8947 & 4.1231 & 0.8785 & 4.0975 & 1.8 \\
\hline \multirow{6}{*}{$\mathrm{N}_{12}=9$} & 0.1 & 2.1218 & 2.5704 & 2.1132 & 2.6043 & 1.3 \\
\hline & 0.5 & 1.5569 & 2.7503 & 1.5392 & 2.7986 & 1.7 \\
\hline & 1.0 & 1.3951 & 3.1717 & 1.3730 & 3.2205 & 1.6 \\
\hline & 2.0 & 1.3056 & 3.9485 & 1.2797 & 3.9776 & 2.0 \\
\hline & 5.0 & 1.3733 & 5.8511 & 1.3423 & 5.8099 & 2.2 \\
\hline & 10.0 & 1.6931 & 8.5317 & 1.6521 & 8.3860 & 2.4 \\
\hline \multirow{6}{*}{$\mathrm{N}_{12}=0.11$} & 0.1 & 2.1302 & 2.0583 & 2.1134 & 2.0562 & 0.8 \\
\hline & 0.5 & 1.3709 & 1.6547 & 1.3415 & 1.6450 & 2.1 \\
\hline & 1.0 & 1.0886 & 1.6080 & 1.0573 & 1.5963 & 2.9 \\
\hline & 2.0 & 0.8537 & 1.6867 & 0.8250 & 1.6728 & 3.4 \\
\hline & 5.0 & 0.6675 & 2.1234 & 0.6481 & 2.1106 & 2.9 \\
\hline & 10.0 & 0.6805 & 2.9516 & 0.6683 & 2.9418 & 1.8 \\
\hline
\end{tabular}
mum absolute deviation of the outputs of the BGK model from those obtained by the McCormack one. 
the Poiseuille flow rates $Q^{(s)}(s=1,2)$ for the two mixtures under investigation. The analysis has been carried out for a wide range of the rarefaction parameter (progressing from free molecular, through transitional, to continuum regions) and for three different values of the ratio of the molar concentrations of the two species $N_{12}=n_{1,0} / n_{2,0}=0.11,1,9$. To make the comparison with the data available in the literature more straightforward, we have reported in Tables I-III the values of the mass flow rate as a function of a rarefaction parameter $\delta^{*}$ according to the definition used in Ref. 18. The relationship between $\delta^{*}$ and the analogous parameter $\delta$ introduced in Sec. III is given by

$$
\delta=\delta^{*} \frac{\left[1+\Theta_{12} N_{12}\right]}{\left[\Theta_{12}\left(1+N_{12}\right)\right]}\left[\frac{M_{12}\left(1+N_{12}\right)}{\left(1+N_{12} M_{12}\right)}\right]^{1 / 2} .
$$

As revealed by these tables, the agreement between the outputs of the BGK and the McCormack models for intermolecular rigidsphere interactions is very good: the maximum absolute deviation between the results of the two models, defined by

$$
\Delta_{\max }=: \max _{s}\left[100\left|\frac{Q_{B G K}^{(s)}}{Q_{M c C}^{(s)}}-1\right|\right],
$$

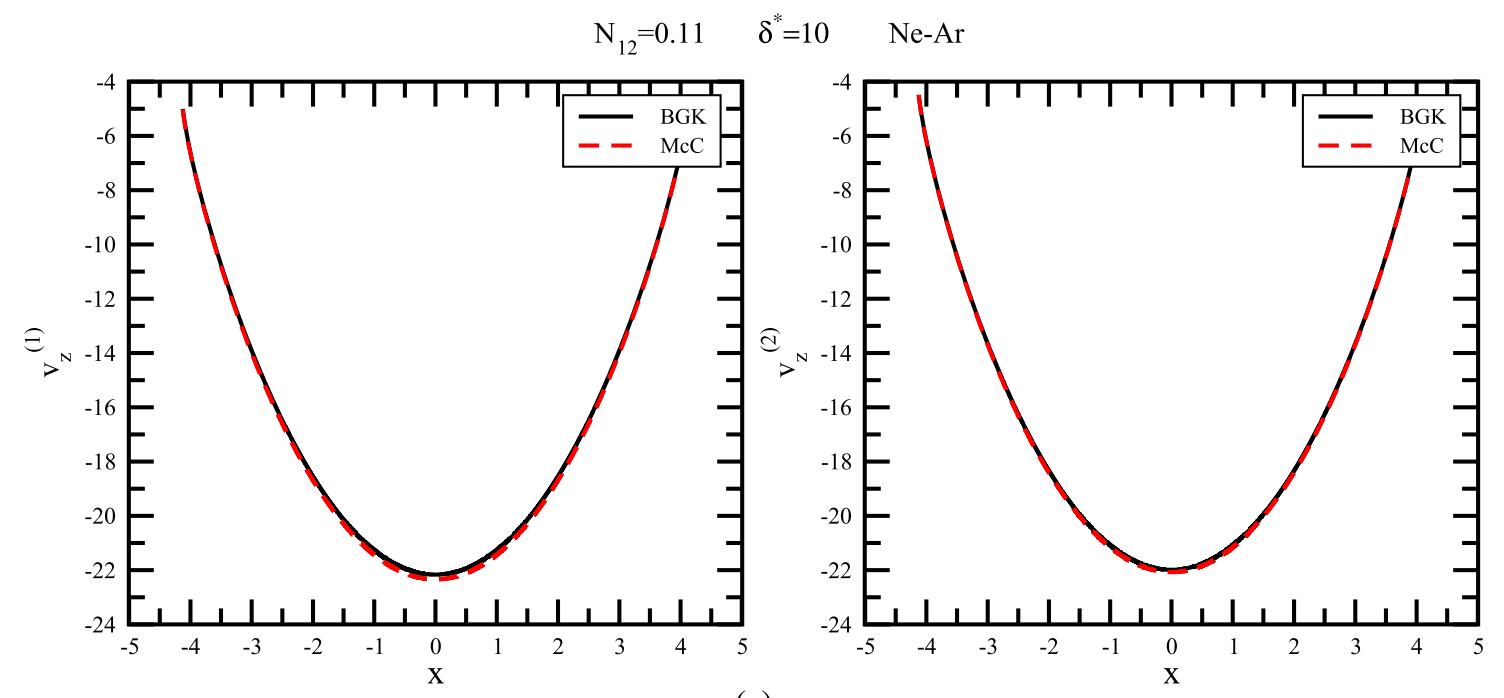

(a)

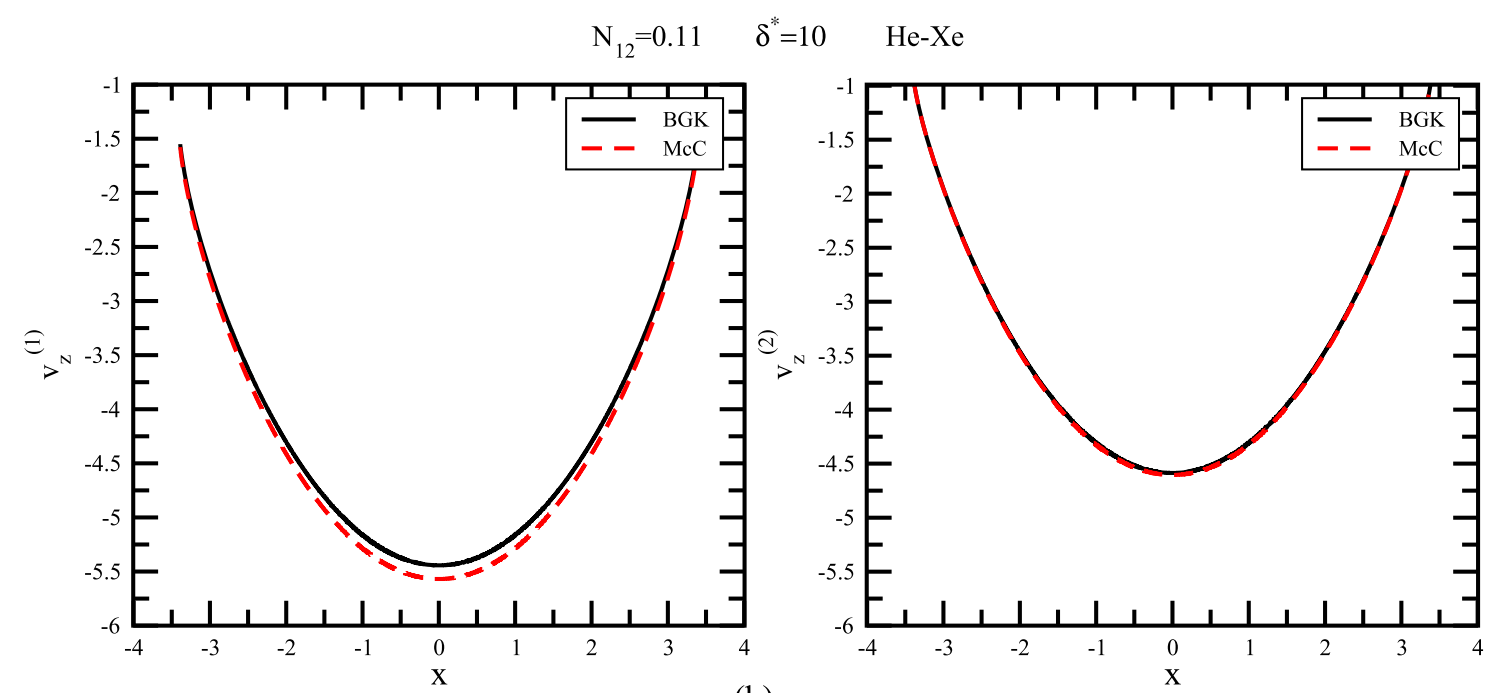

(b)

FIG. 1. Variation of the macroscopic velocities of the (a) Ne-Ar and (b) He-Xe mixture components, in the $x$-direction across the gap of the channel, for $N_{12}=0.11$ and $\delta^{*}=10$. Comparison between the modified BGK model (solid lines) and the McCormack model (dashed lines) for hard-sphere interactions. 
is less than $5 \%$, in all cases considered. The largest difference in percentage is observed for the $\mathrm{He}-\mathrm{Xe}$ mixture, in the transitional regime, regardless of the value of the molar concentrations ratio of the two species. In Table I, we have also reported the findings obtained from a DSMC method designed to solve the true linearized Boltzmann equation for binary gas mixtures of hardsphere molecules. ${ }^{31}$ The comparison shows a basic good agreement between the three approaches presented, proving that the modified BGK model, proposed in the present paper, provides outputs very close to those given by more refined kinetic descriptions, when the same intermolecular potential is considered. On the contrary, the BGK model derived in closed-form for Maxwell molecules in Ref. 9 (see also Ref. 25) reveals larger deviations from the McCormack model, especially for the He-Xe mixture, as highlighted in Tables II and III. Unfortunately, the BGK model, by construction, cannot accurately reproduce the profiles of higher-order moments, such as the heat flux, just as it happens for single monatomic gases. In order to prove more clearly that the modified BGK model, proposed in the present paper, can be applied with general collision kernels and that it gives always outputs very close to the McCormack model, provided that the same intermolecular potential is considered, we report in Table IV also the values of the Poiseuille flow rates $Q^{(s)}$ corresponding to the (6-12) LennardJones (LJ) molecular model. Since the effect of using different interaction potentials is more evident in disparate-mass gas mixtures, Table IV shows only the findings related to the $\mathrm{He}-\mathrm{Xe}$

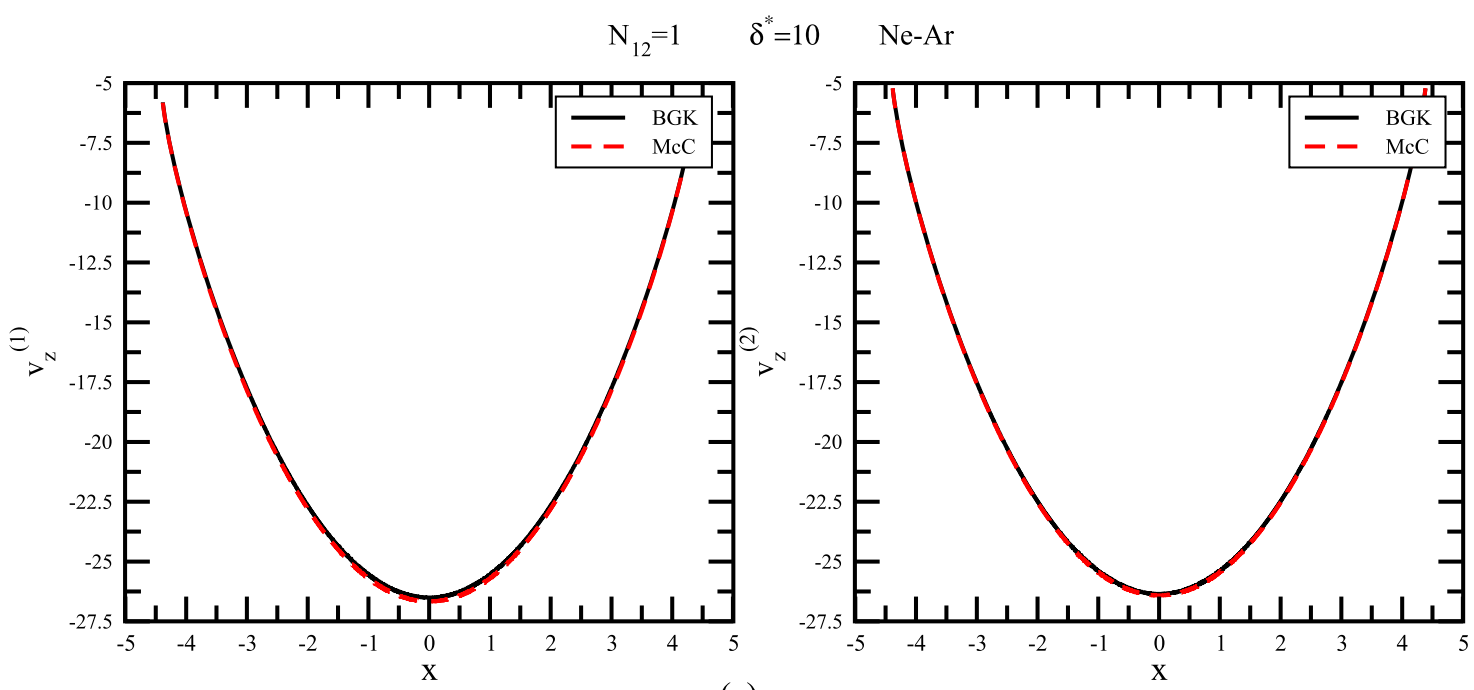

(a)

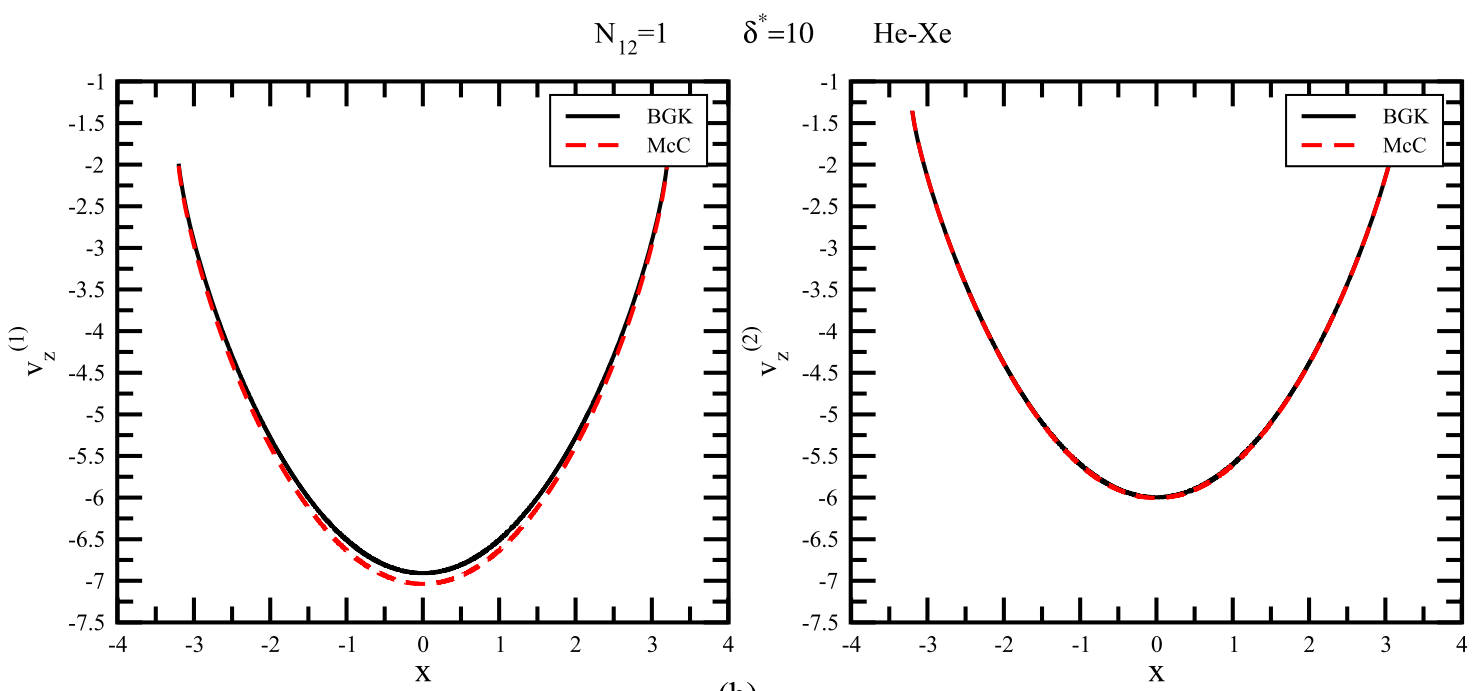

(b)

FIG. 2. Variation of the macroscopic velocities of the (a) Ne-Ar and (b) He-Xe mixture components, in the $x$-direction across the gap of the channel, for $N_{12}=1$ and $\delta^{*}=10$. Comparison between the modified BGK model (solid lines) and the McCormack model (dashed lines) for hard-sphere interactions. 
mixture. In this table, the values related to the McCormack model have been obtained by numerically solving the problem described in Appendix B [Eqs. (B1)-(B4)] with diffuse reflection boundary conditions through a deterministic finite-difference method. ${ }^{33}$ The results reported in Ref. 17 have been used to validate our numerical code.

In order to inspect more deeply the physical processes going on in both gas mixtures, we report in Figs. 1-6 the velocity profile of the $\mathrm{Ne}-\mathrm{Ar}$ and $\mathrm{He}-\mathrm{Xe}$ mixture components as a function of the distance across the gap of the channel, specializing the analysis to rigid-sphere interactions. We have included in these pictures the solutions of the integral equations (47) and (48) in the two opposite regions of rarefaction: the near-continuum regime $\left(\delta^{*}=10\right)$ and the near-free molecular flow regime $\left(\delta^{*}=0.1\right)$, for three different values of the ratio of the molar concentrations of the two species $\left(N_{12}=0.11\right.$, 1 , and 9). To further validate the reliability of our semi-analytical results, we have also reported in Figs. 1-6 the outcomes derived from a numerical solution of the linearized McCormack model. In all the pictures presented, the lightest component of each mixture has been labeled with superscript 1 , while the heaviest one with superscript 2 .

\section{A. Near-continuum regime}

Concerning the Ne-Ar mixture, Figs. 1-3 reveal that, in the near-continuum limit, both species have the same macroscopic velocity fields. On the contrary, for the $\mathrm{He}-\mathrm{Xe}$ mixture, two

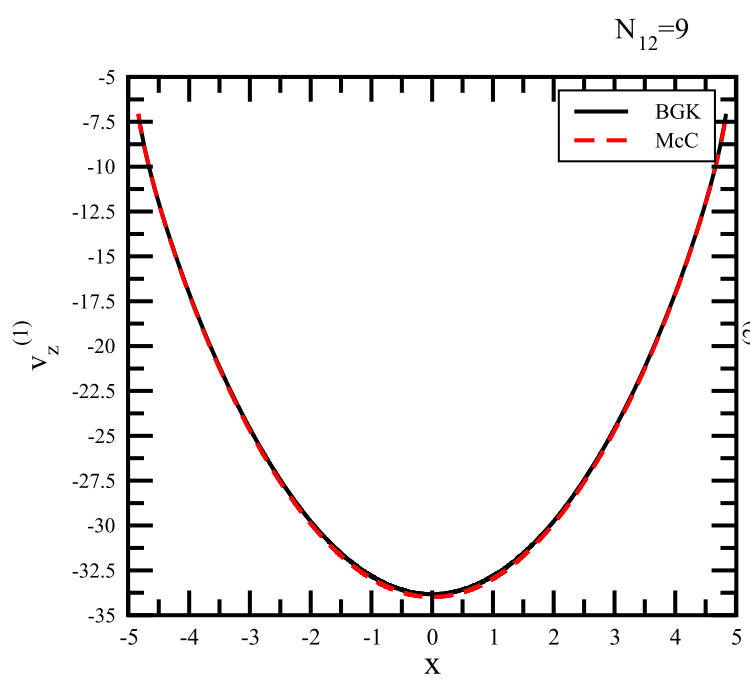

$\delta^{*}=10 \quad \mathrm{Ne}-\mathrm{Ar}$

(a)

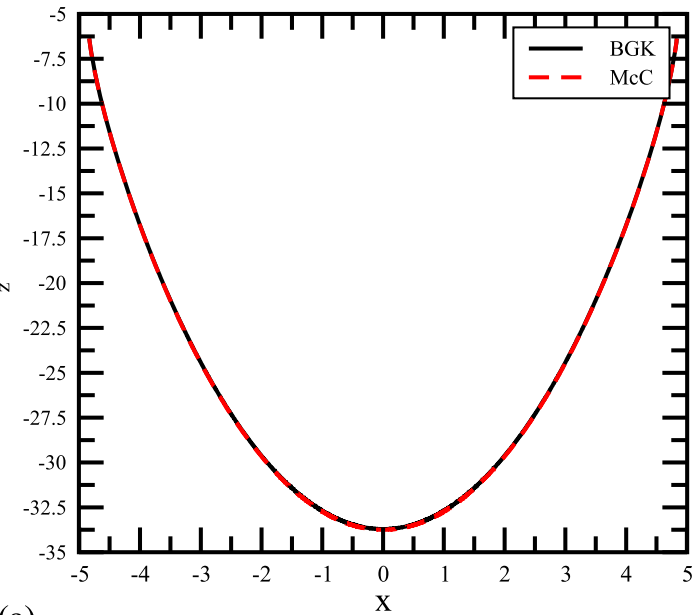

$\mathrm{N}_{12}=9 \quad \delta^{*}=10 \quad \mathrm{He}-\mathrm{Xe}$
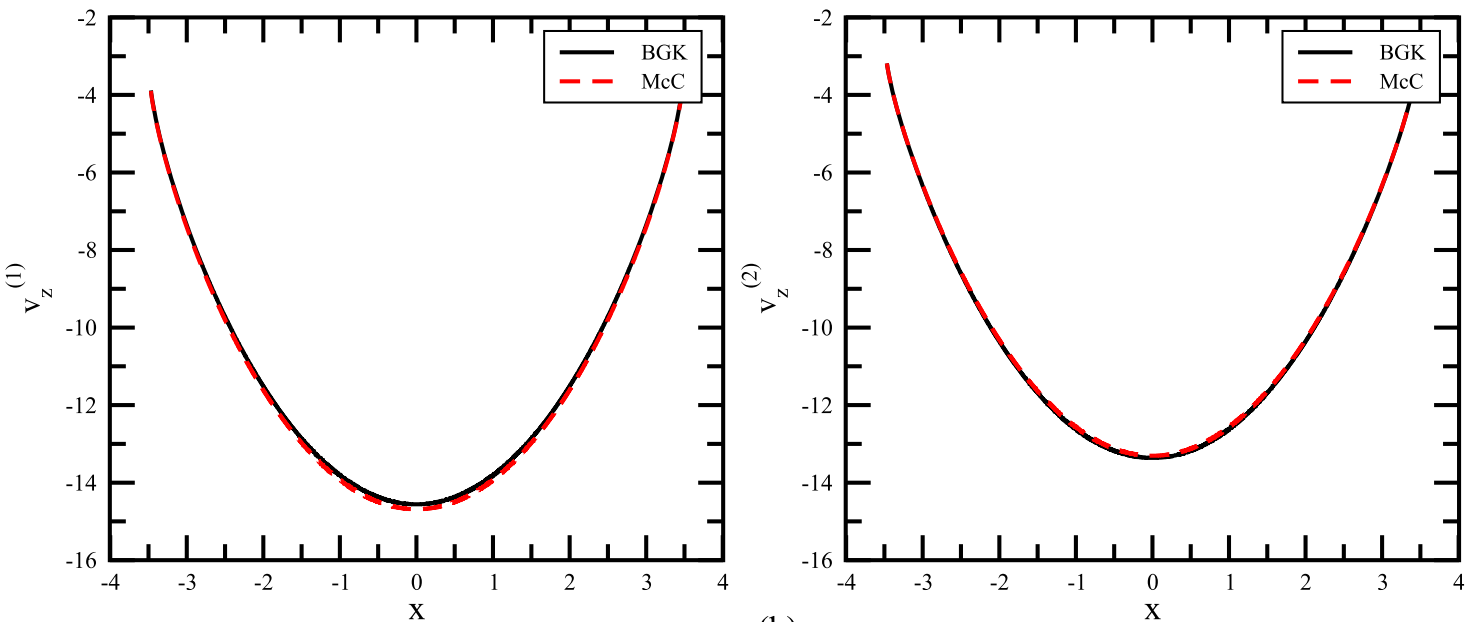

(b)

FIG. 3. Variation of the macroscopic velocities of the (a) Ne-Ar and (b) He-Xe mixture components, in the $x$-direction across the gap of the channel, for $N_{12}=9$ and $\delta^{*}=10$ Comparison between the modified BGK model (solid lines) and the McCormack model (dashed lines) for hard-sphere interactions. 


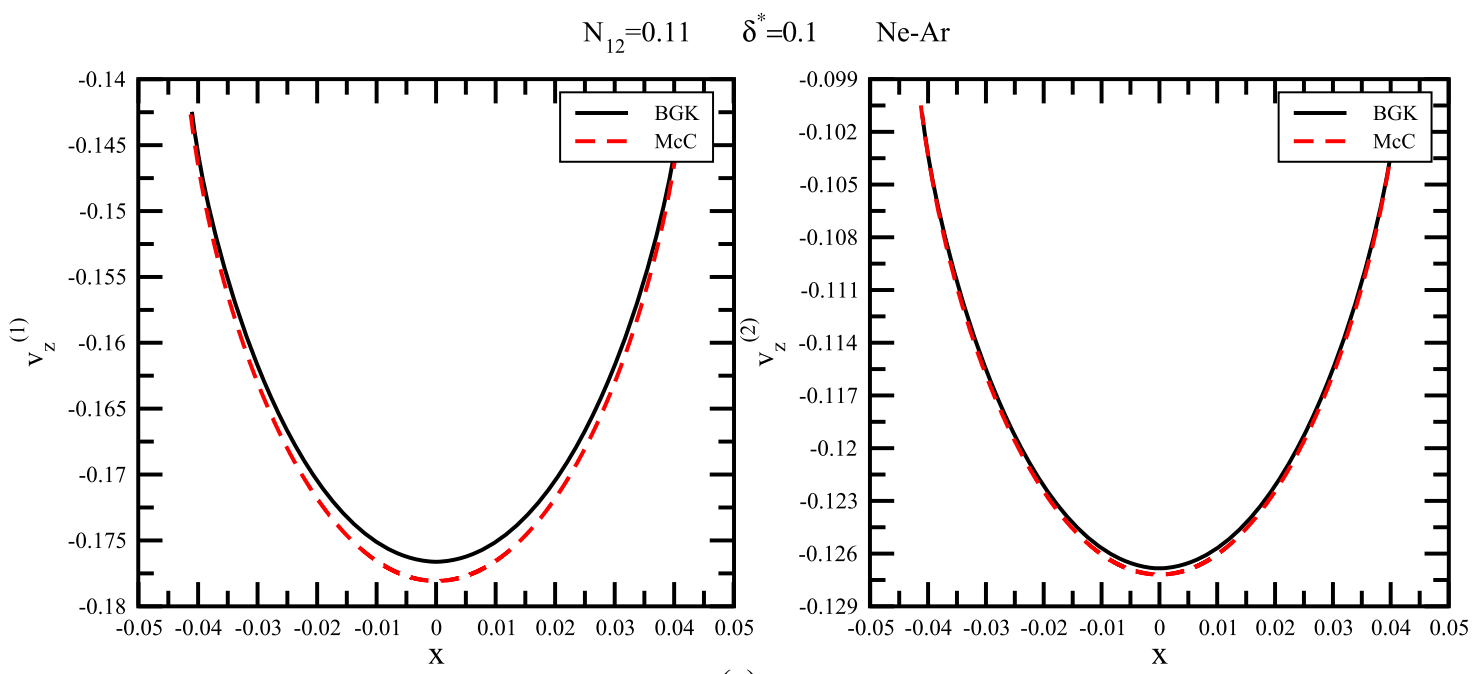

(a)

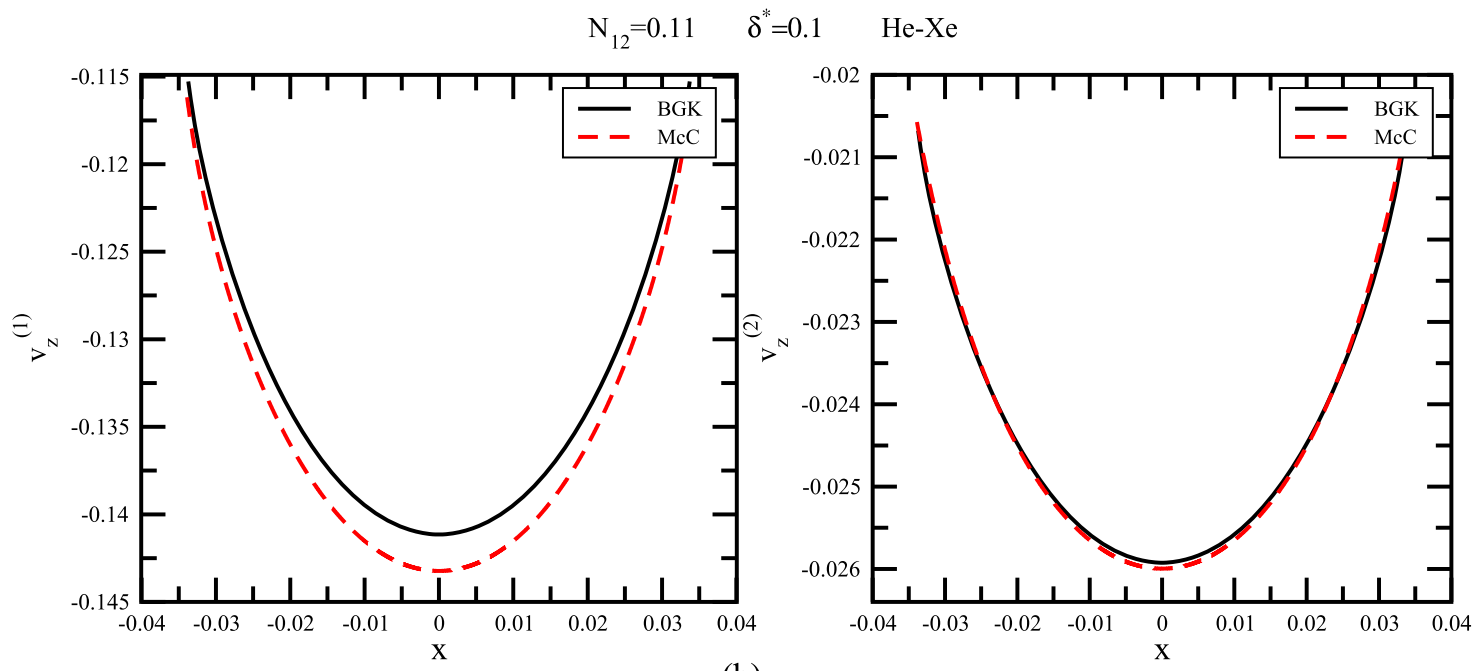

(b)

FIG. 4. Variation of the macroscopic velocities of the (a) Ne-Ar and (b) He-Xe mixture components, in the $x$-direction across the gap of the channel, for $N_{12}=0.11$ and $\delta^{*}=0.1$. Comparison between the modified BGK model (solid lines) and the McCormack model (dashed lines) for hard-sphere interactions.

different velocity profiles are shown. This contrasting behavior of the two mixtures is related to their different relaxation times to equilibrium. As already pointed out in Ref. 25, the analysis of the gas-mixture equations is more difficult than the comparable single component gas theory because of the many different scales which now enter in the approach to equilibrium. There is the approach of the distribution function to a Maxwellian distribution, and in addition, there is the equilibration of the species (i.e., the vanishing of differences in velocity and temperature among the species). In the sixties, Grad conjectured that when a binary gas mixture is made up of species with very different molecular masses, the approach of the gas to equilibrium should exhibit very different relaxation times. ${ }^{38}$ First, the light species and then the heavy species reach approximate local Maxwell equilibrium, in times of the orders of the different species self-collision times: $\theta_{1}$ for the light-species and $\theta_{2}$ for the heavy species. Equilibration between the species can only be attained thereafter on a time scale of order $\theta_{\Delta}$. When one assumes comparable number densities $n_{1} \simeq n_{2}$, the times characterizing these different epochs are expected to stand in the ratios ${ }^{38}$

$$
\frac{\theta_{1}}{\theta_{2}} \simeq \frac{\theta_{2}}{\theta_{\Delta}} \simeq\left(\frac{m_{1}}{m_{2}}\right)^{1 / 2},
$$

with $m_{1}$ and $m_{2}$ being the molecular masses of the light and heavy species, respectively. If $\left(m_{1} / m_{2}\right)$ is very small, as it happens for disparate-mass gas mixtures, then $\theta_{\Delta}$ becomes of the order of a typical macroscopic time. One can easily prove that Grad conjecture (65) is satisfied by the BGK model proposed in the present 


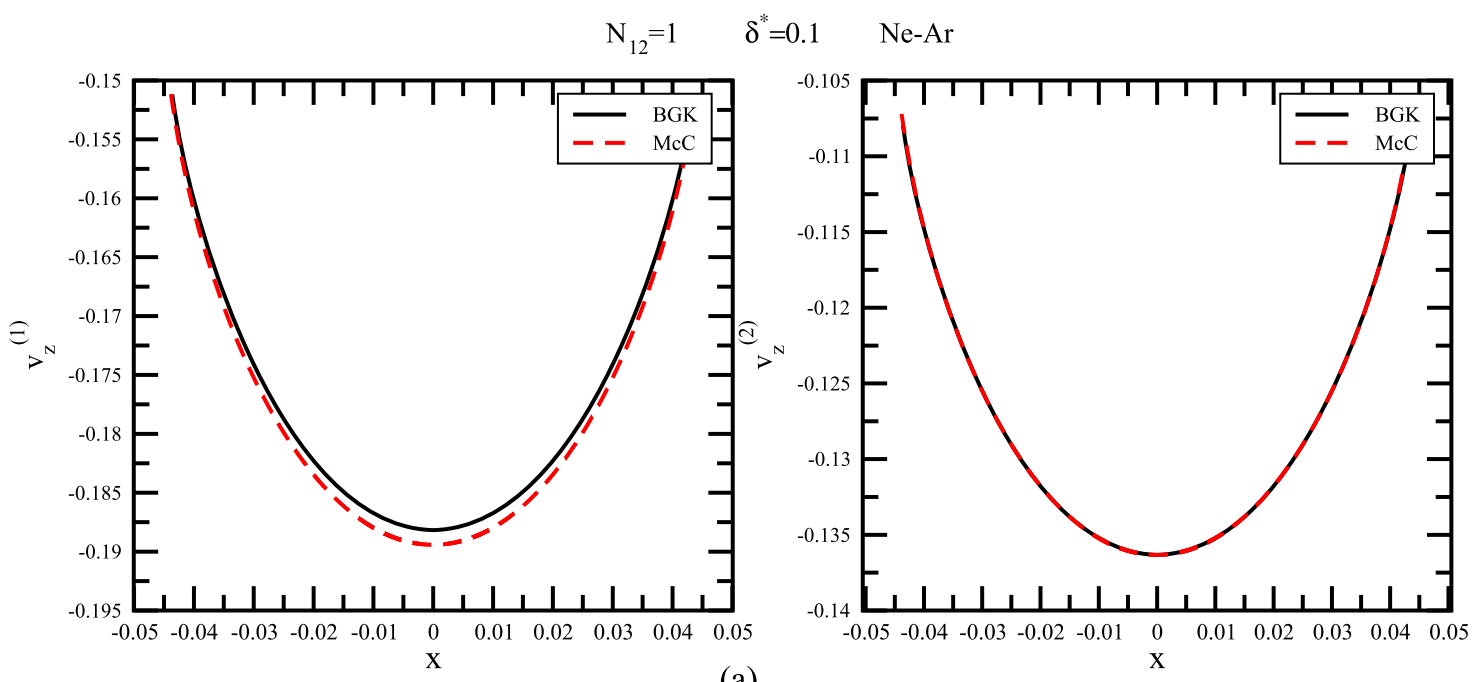

(a)

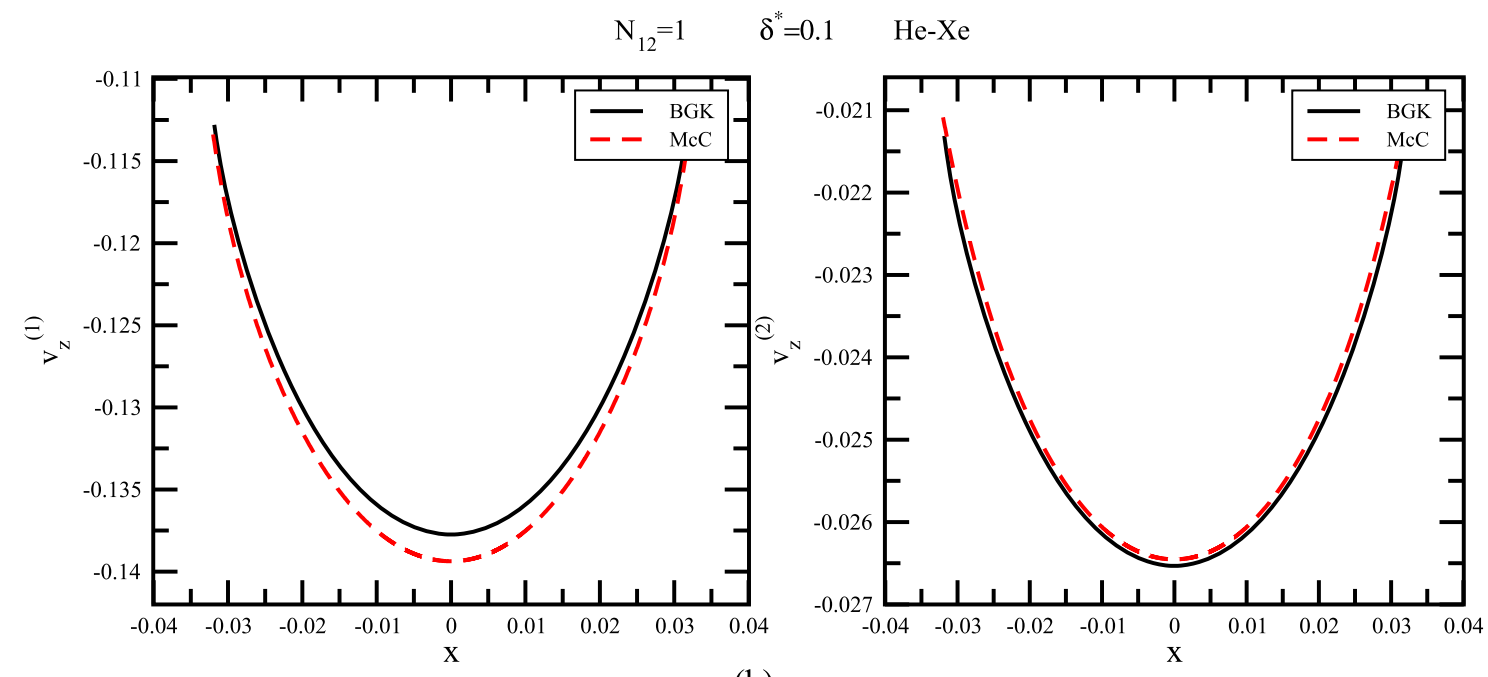

(b)

FIG. 5. Variation of the macroscopic velocities of the (a) Ne-Ar and (b) He-Xe mixture components, in the $x$-direction across the gap of the channel, for $N_{12}=1$ and $\delta^{*}=0.1$. Comparison between the modified BGK model (solid lines) and the McCormack model (dashed lines) for hard-sphere interactions.

paper, using Eq. (13) to calculate $\Theta_{12}=\theta_{1} / \theta_{2}=\gamma_{2} / \gamma_{1}$. This feature is shared with the linearized McCormack model presented in Sec. II A, whose outputs have recently been compared with flow rate measurements under isothermal conditions, showing good agreement with the experimental data. ${ }^{27}$ Therefore, one can conclude that the reliability of the results provided by the BGK model is mainly related to its ability to correctly reproduce the trend to equilibrium of a binary gaseous mixture. In the light of these remarks, the greater complexity of the McCormack model is not completely justified, except in relation to its ability to appropriately describe higher-order moments of the distribution function (as the heat-flow rate).

To explain in more detail the different behavior of the two mixtures, we can resort to a qualitative analysis of the integral equations given by Eqs. (47) and (48). In the near-continuum regime, the integrals in Eqs. (47) and (48) give the main contribution to the velocities of gas components. For mixtures such as $\mathrm{Ne}-\mathrm{Ar}$, whose constituents have comparable molecular mass, such that $\Theta_{12} \simeq 1$ and $M_{12} \simeq 1$, the velocity profiles of the two species are symmetric by interchanging superscripts 1 and 2 (as the results reported in Figs. 1-3 show). In this case, Eq. (47) reduces to the equation for the velocity field of a single-component gas since the terms $\left(-\eta_{1,2}^{(1)} v_{z}^{(1)}\right)$ and $\left(\eta_{1,2}^{(1)} v_{z}^{(2)}\right)$ cancel out. On the contrary, for mixtures such as $\mathrm{He}-\mathrm{Xe}$, whose constituents have very different molecular mass, such that $\Theta_{12} \ll 1$ and $M_{12} \ll 1$, the velocity profiles of the two species are not symmetric anymore (see Figs. 1-3). In this case, Eq. (47) reduces to the equation for the velocity 


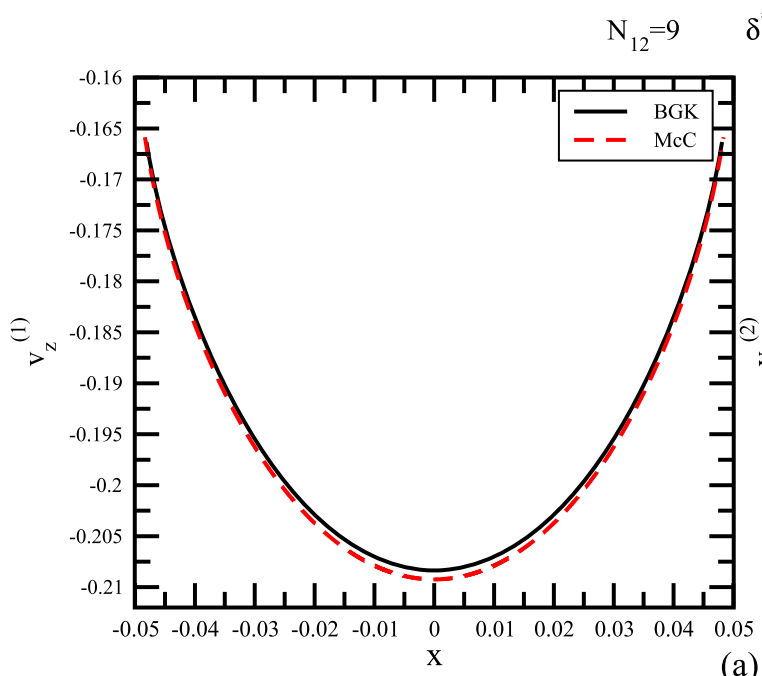

$\delta^{*}=0.1 \quad \mathrm{Ne}-\mathrm{Ar}$

(a)

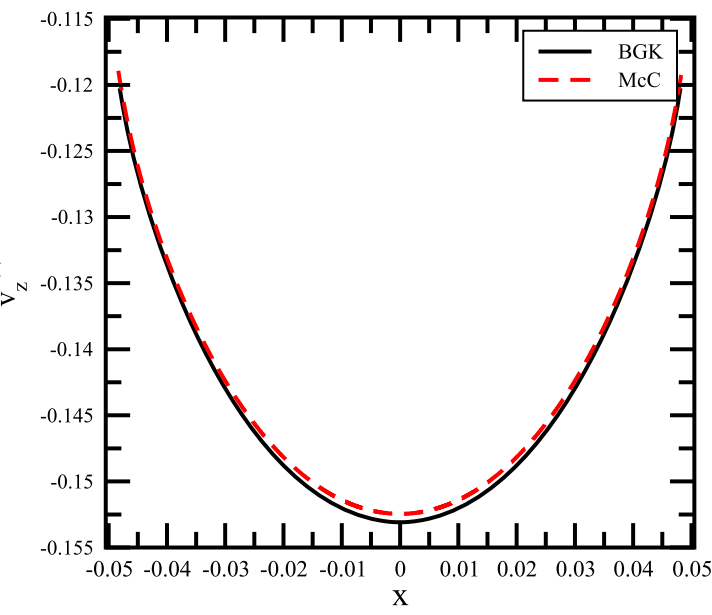

$\mathrm{N}_{12}=9 \quad \delta^{*}=0.1 \quad \mathrm{He}-\mathrm{Xe}$
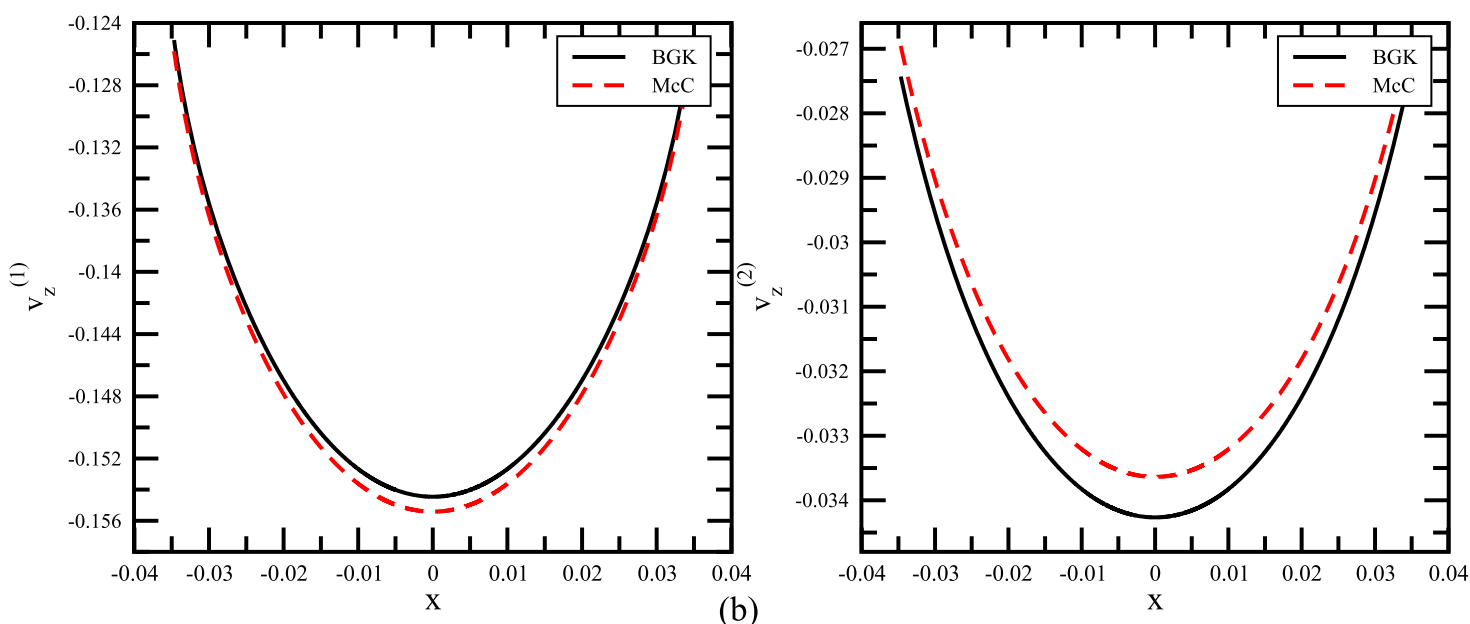

FIG. 6. Variation of the macroscopic velocities of the (a) Ne-Ar and (b) He-Xe mixture components, in the $x$-direction across the gap of the channel, for $N_{12}=9$ and $\delta^{*}=0.1$. Comparison between the modified BGK model (solid lines) and the McCormack model (dashed lines) for hard-sphere interactions.

field of a single-component gas only when $\eta_{1,2}^{(1)} \rightarrow 0$, that is, for $N_{1,2} \gg 1$.

\section{B. Near-free molecular flow regime}

In the near-free molecular flow regime, the integrals appearing in Eqs. (47) and (48) vanish. For mixtures such as Ne-Ar, whose constituents have comparable molecular mass $\left(\Theta_{12} \simeq 1\right.$ and $\left.M_{12} \simeq 1\right)$, the macroscopic velocity fields $v_{z}^{(1)}$ and $v_{z}^{(2)}$ are close to each other, while a greater discrepancy is predicted by formulas (47) and (48) for the velocity profiles of disparate-mass gas mixtures $\left(\Theta_{12}\right.$ $\ll 1$ and $\left.M_{12} \ll 1\right)$. This trend is clearly observed in Figs. 4-6. Moreover, Eq. (47) predicts that, in the near-free molecular flow regime, the macroscopic field profiles of the lightest species of both mixtures coincide with those obtained for a single gas, regardless of the value $N_{12}$ taken by the ratio of the molar concentrations of the two species.

\section{CONCLUDING REMARKS}

In the present paper, we have analyzed the properties of a BGK-type collision operator of the linearized Boltzmann equation designed to describe mixtures of monatomic gases. We have shown that this BGK model, originally derived in closed form for Maxwell molecules in Ref. 9, can be modified (in its linearized form) to take into account general and more realistic intermolecular force laws. In order to assess the reliability of this modified BGK model, we have studied the Poiseuille problem, that is, the flow of a binary gas mixture through a microchannel, driven by a 
small pressure gradient. Semi-analytical solutions of the linearized BGK-Boltzmann equations have been presented, and the results are compared with those derived by the direct simulation Monte Carlo (DSMC) approach. ${ }^{31,32}$ In particular, we have considered two different datasets: one obtained from a classical DSMC method, designed to solve the true linearized Boltzmann equation for binary gas mixtures with hard-sphere molecules, and the other derived from a variance-reduced direct simulation Monte Carlo method, specific for low speed flows, as defined by the McCormack kinetic model.

The variance reduction ideas, presented first in Ref. 39, have effectively addressed the limitation suffered by the standard DSMC method for low Mach number flows, allowing the simulation of arbitrarily small deviations from equilibrium. While, originally, the variance reduction formulation has been used to solve the Boltzmann equation for single component gases, ${ }^{40}$ in more recent years, these ideas have been applied to construct an efficient algorithm for handling simplified kinetic models for mixtures, as the McCormack one. ${ }^{32}$ The outputs of this variance-reduced DSMC method, designed to solve the McCormack linearized kinetic model, have been compared with the discrete ordinate solutions of the same kinetic model for different channel flows, ${ }^{18}$ and a good agreement between the two approaches has been found. This proves the reliability of the DSMC computations that can rightly be considered an effective alternative to the experiments. In this framework, the modified BGK model proposed in the present paper, which is described by a set of equations much simpler than the ones the McCormack model uses (allowing also a semi-analytical representation of the solution), can be helpful to improve the computational efficiency of the DSMC scheme. This is basically due to the fact that, in the simplified description offered by the BGK model, the influence of the main parameters which determine the macroscopic behavior of a binary mixture (as the time scales characterizing the approach to the equilibrium and the microscopic collision frequencies responsible for the deviation from the single-gas equations) can easily be assessed.

\section{ACKNOWLEDGMENTS}

The author was supported by the GNFM of Indam, Italy.

\section{APPENDIX A: COMPUTATION OF THE CHAPMAN-COWLING INTEGRALS}

In the following, we report the explicit expressions of the $\Omega_{s, r}^{i j}$ integrals for the intermolecular interaction potentials taken into account in the present paper.

\section{Maxwell molecules}

The Maxwell molecular model represents an important special case of the inverse power-law interaction potential,

$$
U^{s r}=\frac{K^{s r}}{r^{v-1}},
$$

when $v=5$. In (A1), $r$ is the distance between the center of the molecules and $K^{s r}$ is the interparticle force law constant. In this case, the $\Omega$-integrals are given by ${ }^{14}$

$$
\Omega_{s, r}^{i j}=\frac{A_{i}(5)}{2} \sqrt{\frac{\pi K^{s r}}{\mu^{s r}}} \Gamma\left(j+\frac{3}{2}\right),
$$

where the symbol $\Gamma$ denotes the gamma function, while the constants $A_{i}(5)$ represent the dimensionless collision cross sections, whose values are tabulated in Ref. 14 . The constants $K^{11}$ and $K^{22}$ can be written in terms of the single gas viscosity coefficients $\eta_{s}(s=1,2)$ with the aid of the first Chapman-Enskog expressions for these quantities,

$$
\eta_{s}=\frac{1}{3 \pi}\left(\frac{2 m_{s}}{K^{s s}}\right)^{1 / 2} \frac{\left(k_{B} T\right)}{A_{2}(5)}
$$

while $K^{12}$ may be determined from the method of the combination rule,

$$
K^{12}=\left(K^{11} K^{22}\right)^{1 / 2} .
$$

In order to specify the force constants $K^{11}$ and $K^{22}$, the experimental data on the viscosities $\eta_{s}$ of the single gases at the temperature $T=300 \mathrm{~K}$ have been used, given in Ref. 37 .

\section{Hard-sphere molecules}

Rigid elastic spherical molecules may be regarded as a limiting case of the inverse power-law potential model (A1) corresponding to $v=\infty$. For this rigid sphere model, the $\Omega$-integrals read

$$
\Omega_{s, r}^{i j}=\frac{(j+1) !}{8}\left[1-\frac{\left(1+(-1)^{i}\right)}{2(i+1)}\right]\left(\frac{\pi k_{B} T_{0}}{2 \mu^{s r}}\right)^{1 / 2}\left(d_{s}+d_{r}\right)^{2},
$$

where $d_{s}$ is the molecular diameter of species $s$. Following Ref. 17, the rigid-sphere diameters $d_{s}(s=1,2)$ of every species $s$ are computed via the expression

$$
\eta_{s}=1.016034 \cdot \frac{5}{16} \frac{\sqrt{m_{s} k_{B} T}}{\sqrt{\pi} d_{s}^{2}}
$$

and the experimental data on the viscosities $\eta_{s}$ of the single gases at the temperature $T=300 \mathrm{~K}$, reported in Ref. 37 .

\section{3. (6-12) Lennard-Jones model}

In this model, the potential of intermolecular force is given by

$$
U^{s r}=4 \epsilon^{s r}\left[\left(\frac{d^{s r}}{r}\right)^{12}-\left(\frac{d^{s r}}{r}\right)^{6}\right]
$$

where $r$ is the distance between the center of the molecules, $\epsilon^{s r}$ is the depth of the potential well (the maximum energy of attraction), and $d^{s r}$ is the reference collision diameter [defined so that $\left.U^{s r}\left(d^{s r}\right)=0\right]$. In this case, the $\Omega$-integrals cannot be evaluated analytically in closed form and numerical integrations have to be used. In the present paper, we consider the results of this numerical work tabulated in Ref. 41, assuming the same temperature as that for the Maxwell molecules and the rigid spheres, i.e., $T=300 \mathrm{~K}$. 


\section{APPENDIX B: POISEUILLE FLOW PROBLEM DESCRIBED BY THE MCCORMACK MODEL}

In order to obtain the McCormack model equations describing flows of binary mixtures between two parallel plates driven by pressure gradients, we multiply Eq. (34) [based on the McCormack-collision operator as defined in Eqs. (9) and (10)] by $\frac{1}{\pi} c_{z} e^{-\left(c_{y}^{2}+c_{z}^{2}\right)}$, when $s=1$, and by $\frac{1}{\pi M_{12}^{3 / 2}} c_{z} e^{-\left(c_{y}^{2}+c_{z}^{2}\right) / M_{12}}$, when $s=2$, and we integrate over all $c_{y}$ and $c_{z}$. Then, we multiply Eq. (34) by $\frac{1}{\pi}\left(c_{y}^{2}+c_{z}^{2}-2\right) c_{z} e^{-\left(c_{y}^{2}+c_{z}^{2}\right)}$, when $s=1$, and by $\frac{1}{\pi M_{12}^{3 / 2}}\left[\left(\frac{c_{y}^{2}+c_{z}^{2}}{M_{12}}\right)-2\right] c_{z} e^{-\left(c_{y}^{2}+c_{z}^{2}\right) / M_{12}}$, when $s=2$, and we integrate again over all $c_{y}$ and $c_{z}$. The resulting equations after the projection are

$$
\begin{aligned}
c_{x} \frac{\partial Z^{(1)}}{\partial x}+Z^{(1)}+1= & \left(1-\eta_{1,2}^{(1)}\right) v_{z}^{(1)}-\eta_{1,2}^{(2)} q_{z}^{(1)}+\left(1+\eta_{1,1}^{(4)}-\eta_{1,1}^{(3)}-\eta_{1,2}^{(3)}\right) c_{x} \tilde{P}_{x z}^{(1)} \\
& +\frac{4}{5}\left(1+\eta_{1,1}^{(6)}-\eta_{1,1}^{(5)}-\eta_{1,2}^{(5)}\right)\left(c_{x}^{2}-\frac{1}{2}\right) q_{z}^{(1)}-\frac{1}{2} \eta_{1,2}^{(2)}\left(c_{x}^{2}-\frac{1}{2}\right) v_{z}^{(1)}+\eta_{1,2}^{(1)} v_{z}^{(2)} \\
& +M_{12} \eta_{1,2}^{(2)} q_{z}^{(2)}+\frac{1}{2} \eta_{1,2}^{(2)}\left(c_{x}^{2}-\frac{1}{2}\right) v_{z}^{(2)}+\frac{1}{M_{12}} \eta_{1,2}^{(4)} c_{x} \tilde{P}_{x z}^{(2)}+\frac{4}{5} \frac{\eta_{1,2}^{(6)}}{\sqrt{M_{12}}}\left(c_{x}^{2}-\frac{1}{2}\right) q_{z}^{(2)}, \\
c_{x} \frac{\partial Z^{(2)}}{\partial x}+\Theta_{12} Z^{(2)}+\sqrt{M_{12}}= & \Theta_{12}\left[\frac{1}{\sqrt{M_{12}}}\left(1-\eta_{2,1}^{(1)}\right) v_{z}^{(2)}-\frac{1}{\sqrt{M_{12}}} \eta_{2,1}^{(2)} q_{z}^{(2)}+\frac{1}{\left(M_{12}\right)^{3 / 2}}\left(1+\eta_{2,2}^{(4)}-\eta_{2,2}^{(3)}-\eta_{2,1}^{(3)}\right) c_{x} \tilde{P}_{x z}^{(2)}\right. \\
+ & \frac{4}{5 \sqrt{M_{12}}}\left(1+\eta_{2,2}^{(6)}-\eta_{2,2}^{(5)}-\eta_{2,1}^{(5)}\right)\left(\frac{c_{x}^{2}}{M_{12}}-\frac{1}{2}\right) q_{z}^{(2)}-\frac{\eta_{2,1}^{(2)}}{2 \sqrt{M_{12}}}\left(\frac{c_{x}^{2}}{M_{12}}-\frac{1}{2}\right) v_{z}^{(2)}+\frac{1}{\sqrt{M_{12}}} \eta_{2,1}^{(1)} v_{z}^{(1)} \\
+ & \left.\frac{\eta_{2,1}^{(2)}}{\left(M_{12}\right)^{3 / 2}} q_{z}^{(1)}+\frac{\eta_{2,1}^{(2)}}{2 \sqrt{M_{12}}}\left(\frac{c_{x}^{2}}{M_{12}}-\frac{1}{2}\right) v_{z}^{(1)}+\frac{\eta_{2,1}^{(4)}}{\sqrt{M_{12}}} c_{x} \tilde{P}_{x z}^{(1)}+\frac{4}{5} \eta_{2,1}^{(6)}\left(\frac{c_{x}^{2}}{M_{12}}-\frac{1}{2}\right) q_{z}^{(1)}\right]
\end{aligned}
$$

$$
\begin{gathered}
c_{x} \frac{\partial Y^{(1)}}{\partial x}+Y^{(1)}=-\eta_{1,2}^{(2)} v_{z}^{(1)}+\frac{8}{5}\left(1+\eta_{1,1}^{(6)}-\eta_{1,1}^{(5)}-\eta_{1,2}^{(5)}\right) q_{z}^{(1)} \\
+\eta_{1,2}^{(2)} v_{z}^{(2)}+\frac{8}{5} \frac{\eta_{1,2}^{(6)}}{\sqrt{M_{12}}} q_{z}^{(2)} \\
c_{x} \frac{\partial Y^{(2)}}{\partial x}+\Theta_{12} Y^{(2)}=\Theta_{12}\left[-\frac{\eta_{2,1}^{(2)}}{\sqrt{M_{12}}} v_{z}^{(2)}+\frac{8}{5 \sqrt{M_{12}}}\right. \\
\left.\times\left(1+\eta_{2,2}^{(6)}-\eta_{2,2}^{(5)}-\eta_{2,1}^{(5)}\right) q_{z}^{(2)}+\frac{\eta_{2,1}^{(2)}}{\sqrt{M_{12}}} v_{z}^{(1)}+\frac{8}{5} \eta_{2,1}^{(6)} q_{z}^{(1)}\right],
\end{gathered}
$$

where the reduced unknown distribution functions $Z^{(s)}$ are defined in Eqs. (37) and (38), while the functions $Y^{(s)}$ are given by

$$
\begin{aligned}
& Y^{(1)}\left(x, c_{x}\right)=\frac{2}{\pi \hat{k}} \int_{-\infty}^{+\infty} \int_{-\infty}^{+\infty} h_{1}(x, \mathbf{c}) c_{z}\left(c_{y}^{2}+c_{z}^{2}-2\right) e^{-\left(c_{y}^{2}+c_{z}^{2}\right)} d c_{y} d c_{z}, \\
& Y^{(2)}\left(x, c_{x}\right)= \frac{2}{\pi \hat{k} M_{12}^{3 / 2}} \int_{-\infty}^{+\infty} \int_{-\infty}^{+\infty} h_{2}(x, \mathbf{c}) c_{z} \\
& \times\left[\left(\frac{c_{y}^{2}+c_{z}^{2}}{M_{12}}\right)-2\right] e^{-\left(c_{y}^{2}+c_{z}^{2}\right) / M_{12}} d c_{y} d c_{z} .
\end{aligned}
$$

The macroscopic fields appearing on the right-hand side of Eqs. (B1)-(B4) read as

$$
v_{z}^{(1)}(x)=\frac{1}{\sqrt{\pi}} \int_{-\infty}^{+\infty} Z^{(1)} e^{-c_{x}^{2}} d c_{x}
$$

$$
\begin{gathered}
v_{z}^{(2)}(x)=\frac{1}{\sqrt{\pi}} \int_{-\infty}^{+\infty} Z^{(2)} e^{-c_{x}^{2} / M_{12}} d c_{x} \\
q_{z}^{(1)}(x)=\frac{1}{2 \sqrt{\pi}} \int_{-\infty}^{+\infty}\left[\left(c_{x}^{2}-\frac{1}{2}\right) Z^{(1)}+Y^{(1)}\right] e^{-c_{x}^{2}} d c_{x} \\
q_{z}^{(2)}(x)=\frac{1}{2 \sqrt{\pi}} \int_{-\infty}^{+\infty}\left[\left(\frac{c_{x}^{2}}{M_{12}}-\frac{1}{2}\right) Z^{(2)}+Y^{(2)}\right] e^{-c_{x}^{2} / M_{12}} d c_{x} \\
\tilde{P}_{x z}^{(1)}(x)=\frac{1}{\sqrt{\pi}} \int_{-\infty}^{+\infty} c_{x} Z^{(1)} e^{-c_{x}^{2}} d c_{x} \\
\tilde{P}_{x z}^{(2)}(x)=\frac{1}{\sqrt{\pi}} \int_{-\infty}^{+\infty} c_{x} Z^{(2)} e^{-c_{x}^{2} / M_{12}} d c_{x}
\end{gathered}
$$

\section{REFERENCES}

${ }^{1}$ N. A. Downie, Industrial Gases (Kluwer Academic Publishers, 2002).

${ }^{2} \mathrm{P}$. Tabeling, Introduction to Microfluidics (Oxford University Press, 2011).

${ }^{3}$ C. Cercignani, The Boltzmann Equation and its Applications (Springer, New York, 1988).

${ }^{4}$ E. P. Gross and M. Krook, "Model for collision processes in gases: Smallamplitude oscillations of charged two-component systems," Phys. Rev. 102, 593 (1956).

${ }^{\mathbf{5}}$ L. Sirovich, "Kinetic modeling of gas mixtures," Phys. Fluids 5, 908 (1962).

${ }^{6}$ B. B. Hamel, “Kinetic model for binary gas mixtures," Phys. Fluids 8, 418 (1965). 
${ }^{7}$ E. Goldman and L. Sirovich, "Equations for gas mixtures," Phys. Fluids 10, 1928 (1967).

${ }^{8}$ F. J. McCormack, "Construction of linearized kinetic models for gaseous mixtures and molecular gases,” Phys. Fluids 16, 2095 (1973).

${ }^{9} \mathrm{P}$. Andries, K. Aoki, and B. Perthame, "A consistent BGK-type model for gas mixtures," J. Stat. Phys. 106, 993 (2002).

${ }^{10} \mathrm{~V}$. K. Gupta, H. Struchtrup, and M. Torrilhon, "Regularized moment equations for binary gas mixtures: Derivation and linear analysis," Phys. Fluids 28, 042003 (2016).

${ }^{11}$ R. Gatignol and C. Croizet, "Asymptotic modeling of thermal binary monatomic gas flows in plane microchannels-Comparison with DSMC simulations," Phys. Fluids 29, 042001 (2017).

${ }^{12}$ A. V. Bobylev, M. Bisi, M. Groppi, G. Spiga, and I. F. Potapenko, "A general consistent BGK model for gas mixtures," Kinet. Relat. Models 11, 1377 (2018).

${ }^{13}$ M. Sabouri and M. Darbandi, "Numerical study of species separation in rarefied gas mixture flow through micronozzles using DSMC," Phys. Fluids 31, 042004 (2019).

${ }^{14}$ S. Chapman and T. G. Cowling, The Mathematical Theory of Non-Uniform Gases (Cambridge University Press, 1970).

${ }^{15}$ F. Sharipov and D. Kalempa, "Velocity slip and temperature jump coefficients for gaseous mixtures. I. Viscous slip coefficient,” Phys. Fluids 15, 1800 (2003).

${ }^{16} \mathrm{~F}$. Sharipov and D. Kalempa, "Velocity slip and temperature jump coefficients for gaseous mixtures. II. Thermal slip coefficient," Phys. Fluids 16, 759 (2004).

${ }^{17}$ S. Naris, D. Valougeorgis, D. Kalempa, and F. Sharipov, "Gaseous mixture flow between two parallel plates in the whole range of the gas rarefaction," Physica A 336, 294 (2004).

${ }^{18}$ C. E. Siewert and D. Valougeorgis, "The McCormack model: Channel flow of a binary gas mixture driven by temperature, pressure and density gradients," Eur. J. Mech.: B/Fluids 23, 645 (2004).

${ }^{19} \mathrm{~F}$. Sharipov and D. Kalempa, "Velocity slip and temperature jump coefficients for gaseous mixtures. III. Diffusion slip coefficient," Phys. Fluids 16, 3779 (2004).

${ }^{20}$ S. Naris, D. Valougeorgis, D. Kalempa, and F. Sharipov, "Flow of gaseous mixtures through rectangular microchannels driven by pressure, temperature, and concentration gradients," Phys. Fluids 17, 100607 (2005).

${ }^{21} \mathrm{~S}$. Kosuge and S. Takata, "Database for flows of binary gas mixtures through a plane microchannel,” Eur. J. Mech.: B/Fluids 27, 444 (2008).

${ }^{22}$ P. L. Bhatnagar, E. P. Gross, and M. Krook, "A model for collision processes in gases. I. Small amplitude processes in charged and neutral one-component systems," Phys. Rev. 94, 511 (1954).

${ }^{23}$ C. Cercignani, M. Lampis, and S. Lorenzani, "Variational approach to gas flows in microchannels," Phys. Fluids 16, 3426 (2004).
${ }^{24}$ C. Cercignani, M. Lampis, and S. Lorenzani, "Plane Poiseuille-Couette problem in micro-electro-mechanical systems applications with gas-rarefaction effects," Phys. Fluids 18, 087102 (2006).

${ }^{25} \mathrm{M}$. Bisi and S. Lorenzani, "High-frequency sound wave propagation in binary gas mixtures flowing through microchannels," Phys. Fluids 28, 052003 (2016).

${ }^{26}$ S. Lorenzani, "Kinetic modeling for the time-dependent behavior of binary gas mixtures," in Proceedings of Rarefied Gas Dynamics: 31st International Symposium, 2019.

${ }^{27}$ L. Szalmas, J. Pitakarnnop, S. Geoffroy, S. Colin, and D. Valougeorgis, "Comparative study between computational and experimental results for binary rarefied gas flows through long microchannels,” Microfluid. Nanofluid. 9, 1103 (2010).

${ }^{28}$ C. Cercignani, M. Lampis, and S. Lorenzani, "Flow of a rarefied gas between parallel and almost parallel plates," AIP Conf. Proc. 762, 719 (2005).

${ }^{29}$ C. Cercignani, A. Frangi, S. Lorenzani, and B. Vigna, "BEM approaches and simplified kinetic models for the analysis of damping in deformable MEMS," Eng. Anal. Boundary Elem. 31, 451 (2007).

${ }^{30}$ G. A. Bird, Molecular Gas Dynamics and the Direct Simulation of Gas Flows (Clarendon, Oxford, 1994).

${ }^{31}$ L. Szalmas, "DSMC simulation of binary rarefied gas flows between parallel plates and comparison to other methods," AIP Conf. Proc. 1333, 348 (2011).

${ }^{32}$ L. Szalmas, "Variance-reduced DSMC for binary gas flows as defined by the McCormack kinetic model," J. Comput. Phys. 231, 3723 (2012).

${ }^{33}$ S. Lorenzani, "Semi-analytical methods of solution for the BGK-Boltzmann equation describing sound wave propagation in binary gas mixtures," in Innovative Algorithms and Analysis, edited by L. Gosse and R. Natalini (Springer Indam Series, 2017), Vol. 16, p. 289.

${ }^{34}$ A. B. Huang and D. L. Hartley, "Nonlinear rarefied Couette flow with heat transfer," Phys. Fluids 11, 1321 (1968).

${ }^{35} \mathrm{C}$. Cercignani and A. Daneri, "Flow of a rarefied gas between two parallel plates," J. Appl. Phys. 34, 3509 (1963).

${ }^{36}$ S. K. Loyalka and R. W. Tsai, "Numerical method for solving integral equations of neutron transport," Nucl. Sci. Eng. 58, 193 (1975).

${ }^{37}$ J. Kestin, K. Knierim, E. A. Mason, B. Najafi, S. T. Ro, and M. Waldman, "Equilibrium and transport properties of the noble gases and their mixtures at low density," J. Phys. Chem. Ref. Data 13, 229 (1984).

${ }^{38} \mathrm{H}$. Grad, "Theory of rarefied gases," in Rarefied Gas Dynamics, edited by F. M. Devienne (Pergamon, New York, 1960), pp. 100-138.

${ }^{39}$ L. L. Baker and N. G. Hadjiconstantinou, "Variance reduction for Monte Carlo solutions of the Boltzmann equation," Phys. Fluids 17, 051703 (2005).

${ }^{40}$ T. M. M. Homolle and N. G. Hadjiconstantinou, "Low-variance deviational simulation Monte Carlo," Phys. Fluids 19, 041701 (2007).

${ }^{41}$ J. O. Hirschfelder, C. F. Curtiss, and R. B. Bird, The Molecular Theory of Gases and Liquids (John Wiley \& Sons, New York, 1954). 